\title{
Resveratrol Alleviates 27-Hydroxycholesterol-Induced Senescence in Nerve Cells and Affects Zebrafish Locomotor Behavior via Activation of SIRT1-Mediated STAT3 Signaling
}

\author{
Jiao Liu, ${ }^{1,2}$ Kailin Jiao, ${ }^{1}$ Qian Zhou, ${ }^{1}$ Jun Yang, ${ }^{3}$ Keke Yang, ${ }^{1}$ Chunyan Hu, ${ }^{1}$ Ming Zhou, \\ and Zhong $\mathrm{Li}{ }^{1}{ }^{1}$ \\ ${ }^{1}$ The Key Laboratory of Modern Toxicology of Ministry of Education, School of Public Health, Nanjing Medical University, No. 818 \\ East Tianyuan Rd., Nanjing 211166, China \\ ${ }^{2}$ Department of Nutrition, The Affiliated Suzhou Hospital of Nanjing Medical University, No. 16 West Baita Rd., \\ Suzhou 215000, China \\ ${ }^{3}$ Shenzhen Academy of Metrology \& Quality Inspection, Shenzhen 518131, China
}

Correspondence should be addressed to Zhong Li; lz-ny@njmu.edu.cn

Received 14 December 2020; Revised 9 April 2021; Accepted 2 June 2021; Published 21 June 2021

Academic Editor: Jayeeta Ghose

Copyright (C) 2021 Jiao Liu et al. This is an open access article distributed under the Creative Commons Attribution License, which permits unrestricted use, distribution, and reproduction in any medium, provided the original work is properly cited.

The oxysterol 27-hydroxycholesterol (27HC) is the first identified endogenous selective estrogen receptor modulator (SERM), which like endogenous estrogen $17 \beta$-estradiol $\left(\mathrm{E}_{2}\right)$ induces the proliferation of estrogen receptor- (ER-) positive breast cancer cells in vitro. However, 27HC differs from $\mathrm{E}_{2}$ in that it shows adverse effects in the nervous system. Our previous study confirmed that $27 \mathrm{HC}$ could induce neural senescence by activating phosphorylated signal transducer and activator of transcription, which $\mathrm{E}_{2}$ could not. The purpose of the present study is to investigate whether STAT3 acetylation was involved in 27HC-induced neural senescence. Microglia (BV2 cells) and rat pheochromocytoma cells (PC12 cells) were used in vitro to explore the effect of resveratrol (REV) on 27HC-induced neural senescence. Senescence-associated $\beta$-galactosidase (SA- $\beta$-Gal) staining was performed using an SA- $\beta$-Gal Staining Kit in cells and zebrafish larvae. Zebrafish were used in vivo to assess the effect of $27 \mathrm{HC}$ on locomotor behavior and aging. We found that $27 \mathrm{HC}$ could induce senescence in neural cells, and REV, which has been employed as a Sirtuin-1 (SIRT1) agonist, could attenuate 27HC-induced senescence by inhibiting STAT3 signaling via SIRT1. Moreover, in the zebrafish model, REV attenuated 27HC-induced locomotor behavior disorder and aging in the spinal cord of zebrafish larvae, which was also associated with the activation of SIRT1-mediated STAT3 signaling. Our findings unveiled a novel mechanism by which REV alleviates 27HC-induced senescence in neural cells and affects zebrafish locomotor behavior by activating SIRT1-mediated STAT3 signaling.

\section{Introduction}

Cholesterol can be oxidized in the body by the side chain to form oxidized cholesterol. The side chains contain hydroxyl groups and play multiple roles in lipid metabolism. Among the cholesterol oxidation products, the most abundant and studied category is 27 -hydroxycholesterol (27HC) [1]. The
$27 \mathrm{HC}$ is found almost entirely in the peripheral circulation and can flow into the brain through the blood-brain barrier (BBB). The $27 \mathrm{HC}$ plays an important role in maintaining the balance of brain and extracerebral cholesterol [2]. The $27 \mathrm{HC}$ is a selective estrogen receptor modulator (SERM). The $27 \mathrm{HC}$ functions like endogenous estrogen $17 \beta$-estradiol $\left(E_{2}\right)$ and induces the proliferation of estrogen receptor- (ER-) 
positive breast cancer cells in vitro [3]. Classically, $\mathrm{E}_{2}$ plays a protective role in neurodegenerative diseases. However, it has been reported that $27 \mathrm{HC}$ induces adverse effects in the nervous system, distinguishing it from $\mathrm{E}_{2}$ [4]. This is consistent with our previous study, which confirmed that $27 \mathrm{HC}$ could induce nerve cell senescence as well as tumor invasion and migration but that $\mathrm{E}_{2}$ could not [5-7].

Signal transducer and activator of transcription 3 (STAT3) is a member of the STAT family of transcription factors and is involved extensively in cellular functions such as cell proliferation, cell differentiation, immune response, cell survival, and apoptosis [8-11]. Following receptor activation, Tyr705 phosphorylation of STAT3 induces homo- or heterodimerization of phospho-STAT3 (p-STAT3). Dimerized STAT3 translocates to the nucleus and binds to the promoters bearing cognate DNA-binding sequences [12]. In addition, Lys685 residues of STAT3 can be acetylated by p300 and play a decisive role in the transcriptional activity of STAT3, which stabilizes the dimer formation of STAT3 and is required for activating STAT3 transcription $[13,14]$. Sirtuin-1 (SIRT1), a class III protein deacetylase, potentially plays a role in diabetes, inflammation, and neurodegeneration [15]. It has been reported that SIRT1 counteracts the activation of acetyl-STAT3 (Lys685) and can induce the loss of viability and increase in senescence in gastric cancer (GC) cells [16]. However, the function of SIRT1 and acetyl-STAT3 (Lys685) in 27HC-induced neural senescence and its mechanism remain unclear.

Resveratrol (REV), a polyphenol found in red grapes, red wine, and other plant foods, is receiving increasing attention owing to its medical potential. A recent report showed that REV could increase SIRT1 expression and reduce the senescence-associated secretory phenotype via the SIRT1/NF$\kappa \mathrm{B}$ pathway in the gut of the fish Nothobranchius guentheri [17]. REV has also shown benefit in neurodegenerative diseases, which are a group of chronic, progressive disorders characterized by the gradual loss of neurons in several areas of the central nervous system (CNS) [18].

BV2 cell lines retain a variety of morphology, characterization, and functional characteristics of microglia [19]. PC12 cell has similar morphological, physiological, and biochemical functions with neurons and is widely used in the study of various neurological diseases $[20,21]$. In the present study, using 27HC-treated cells (BV2 and PC12) and zebrafish larvae, we aimed to address the following questions: (1) whether STAT3 acetylation is involved in 27HC-induced neural senescence, (2) whether SIRT1 can regulate STAT3 acetylation by $27 \mathrm{HC}$ treatments, (3) whether reactive oxygen species (ROS) can regulate SIRT1 by methylation following 27HC-exposure, and (4) whether REV can improve 27HCinduced adverse effects.

\section{Materials and Methods}

2.1. Reagents and Cell Culture. The 27HC (purity $\geq 99.9 \%$ ) was purchased from Santa Cruz Biotechnology (Santa Cruz, CA, USA) and was dissolved in absolute ethanol at a stock concentration of $20 \mathrm{mM}$ and then stored at $-80^{\circ} \mathrm{C}$. C646 was purchased from MedChem Express (MCE, USA) and was dissolved in dimethyl sulfoxide (DMSO) at a stock concentration of $10 \mathrm{mM}$ and then stored at $-80^{\circ} \mathrm{C}$. $\mathrm{N}$ Acetylcysteine (NAC) (an antioxidant) was purchased from Beyotime Co., Ltd. (China). REV was purchased from Xuhuang Biotechnology Co., Ltd. (Xi'an, China). Azacitidine (AZA) and S-adenosyl methionine (SAM) were purchased from Sigma-Aldrich. All other reagents were of the highest analytical grade available. Anti-Ac-STAT3 ${ }^{\text {Lys685 }}$ (2523), anti-SIRT1 (8469), anti-DNMT1 (5032), and antip-STAT3 ${ }^{\text {Tyr705 }}$ (9145) were purchased from Cell Signaling Technology (CST, USA).

BV2 microglial cells and PC12 rat pheochromocytoma cells were purchased from the Institute of Basic Medical Sciences of the China Science Academy. The cells were maintained in high glucose Dulbecco's Modified Eagle's Medium (DMEM) supplemented with 10\% fetal bovine serum, 100 units $/ \mathrm{ml}$ penicillin, and $100 \mathrm{mg} / \mathrm{ml}$ streptomycin. They were maintained in a humidified $5 \% \mathrm{CO}_{2} / 95 \%$ air environment at $37^{\circ} \mathrm{C}$.

2.2. Zebrafish Husbandry and Collection of Embryos. $\mathrm{AB}$ strain zebrafish (Danio rerio), purchased from Nanjing EzeRinka Biotechnology Co., Ltd., were kept in a recirculating system at $28.5^{\circ} \mathrm{C}$, with a $14 \mathrm{~h}$ light $/ 10 \mathrm{~h}$ dark cycle 7 days per week [22]. The fish system water was aerated and measured daily to maintain a dissolved oxygen concentration of 7.5-8 mg/l and $\mathrm{pH}$ of 7.0-7.6. The fish were fed with live brine shrimp (Artemia nauplii, Tianjin Ocean Pal Carol Biotech Co., Tianjin, China) twice per day. Male and female adult fish were transferred in pairs at a ratio of $2: 1$ to a spawning aquarium overnight, and the spawning finished during the first $30 \mathrm{~min}$ of the light cycle the next morning. Fertilized eggs were collected, then washed with embryo medium $\left(0.137 \mathrm{M} \mathrm{NaCl}, 5.4 \mathrm{mM} \mathrm{KCl}, 0.25 \mathrm{mM} \mathrm{Na}_{2} \mathrm{HPO}_{4}\right.$, $0.44 \mathrm{mM} \mathrm{KH}_{2} \mathrm{PO}_{4}, 1.3 \mathrm{mM} \mathrm{CaCl}, 1.0 \mathrm{mM} \mathrm{MgSO}$, and $4.2 \mathrm{mM} \mathrm{NaHCO}_{3}$ ) and incubated in Petri dishes at $28 \pm 1^{\circ} \mathrm{C}$ until methamidophos exposure experiments [23]. All experiments were performed with the approval of the Ethics Committee of Nanjing Medical University. All procedures were performed according to the guidelines of the Animal Care Committee of Nanjing Medical University.

\subsection{The 27HC Treatment of Zebrafish Embryos and Larvae.} Test solutions with 27HC were freshly prepared using embryo medium before exposure experiments. The concentrations of the test solutions were 0 (control), $10 \mu \mathrm{M}, 20$ $\mu \mathrm{M}, 30 \mu \mathrm{M}$, or $40 \mu \mathrm{M}$. Zebrafish embryos $36 \mathrm{~h}$ postfertilization (hpf) were transferred to the solutions in a 6-well plate with 30 larvae per well, and the plate was incubated at $28 \pm 1^{\circ} \mathrm{C}$ under the same light cycle as the adults throughout the 4 days postfertilization (dpf) exposure period. The exposure solutions were changed daily to ensure nominal concentrations of $27 \mathrm{HC}$ and water quality.

2.4. Locomotor Behavior Observation. Larvae locomotor activity was monitored at $4 \mathrm{dpf}$ using a Noldus DanioVision (Noldus, Netherlands). Zebrafish embryos 4 dpf were transferred to 96-well plates (one per well). The appropriate amount of test solution per well was used to cover larvae. 
Following a $5 \mathrm{~min}$ habituation period, the locomotor activity was recorded on video for $3 \mathrm{~min}$ [24]. Automated analysis of traces from EthoVision XT software (Noldus, Netherlands) was used to assess distance traveled $(\mathrm{mm})$ and mean speed $(\mathrm{mm} / \mathrm{s})(n=30)[25]$.

2.5. SA- $\beta$-Gal Assay and Quantitation in Cells and Zebrafish Larvae. Senescence-associated $\beta$-galactosidase (SA- $\beta$-Gal) staining was performed using an SA- $\beta$-Gal Staining Kit (Beyotime Co., Ltd.; C0602) according to a standard protocol [26]. SA- $\beta$-Gal-positive cells were considered to be cells with a blue or bright blue color by microscopy. And more than 500 cells in 6 random fields were counted to determine the percentage of SA- $\beta$-Gal-positive cells in the total cells.

Zebrafish larvae were fixed in $4 \%$ paraformaldehyde in phosphate-buffered saline (PBS) at $4^{\circ} \mathrm{C}$ overnight and then washed 3 times for $1 \mathrm{~h}$ in PBS-pH 7.4 and for a further $1 \mathrm{~h}$ in PBS-pH 6.0 at $4^{\circ} \mathrm{C}$. Staining was performed overnight at $37^{\circ} \mathrm{C}$ in $5 \mathrm{mM}$ potassium ferrocyanide, $2 \mathrm{mM} \mathrm{MgCl}_{2}$, and $1 \mathrm{mg} / \mathrm{ml} \mathrm{X-gal} \mathrm{in} \mathrm{PBS} \mathrm{adjusted} \mathrm{to} \mathrm{pH}$ 6.0. All larvae were photographed under the same conditions using reflected light under a dissecting microscope. SA- $\beta$-Gal activity of the neural tube site of zebrafish larvae in each group $(n=30)$ was quantitated using a selection tool in Adobe Photoshop for a color range that was chosen by 25 additive blue color selections of regions that showed visually positive SA- $\beta$-Gal staining. To quantitatively examine SA- $\beta$-Gal levels in vivo, we generated high-resolution digital images that enabled us to select stained pixels using Adobe Photoshop analysis software and then to calculate the percentage of stained pixels out of the net total in each case.

2.6. Detection of ROS Generation in Larvae. For in vivo ROS detection, live 4-dpf-old embryos were incubated with dichlorofluorescein diacetate (DCFH-DA) at $37^{\circ} \mathrm{C}$ for 30 min. The fluorescent signal was observed using a fluorescence microscope. To quantify the ROS levels, the DCFH fluorescence intensity was analyzed by a BD FACSCalibur (BD Biosciences, San Jose, CA, United States) at an excitation wavelength of $488 \mathrm{~nm}$ and an emission wavelength of $525 \mathrm{~nm}$. The experiments were repeated a minimum of three times.

2.7. Western Blot. Total protein was extracted using Radio Immunoprecipitation Assay (RIPA) buffer (Beyotime Co., Ltd.). Then, the protein concentrations were measured with the bicinchoninic acid (BCA) kit (Beyotime Co., Ltd). Afterwards, proteins $(20 \mu \mathrm{g})$ were separated by $10 \%$ sodium dodecyl sulfate-polyacrylamide gel electrophoresis followed by transfer to polyvinylidene fluoride membranes (Millipore, Billerica, USA). After blocking with $10 \%$ nonfat milk, membranes were incubated with the primary antibody at $4^{\circ} \mathrm{C}$ overnight, using antibodies for Ac-STAT3 ${ }^{\text {Lys685 }}$, SIRT1, DNMT1, and p-STAT3 ${ }^{\text {Tyr705 }}$ (CST, 1:500 dilution) and $\beta$-actin (Beyotime Co., Ltd.; 1:500 dilution). Then, the membranes were incubated with horseradish peroxidaseconjugated secondary antibodies (Beyotime Co., Ltd.; 1: 2,000 dilution) for another $1 \mathrm{~h}$ at $37^{\circ} \mathrm{C}$. The immune complexes were detected using an enhanced chemiluminescence kit (CST). Bands were normalized using $\beta$-actin to correct for differences in the loading of the proteins. Densitometric analysis was conducted using Image-Pro Plus 6.0 software as described previously [27].

2.8. Cell Transfection. BV2 and PC12 cells were seeded in 6 -well plates at a density of $1 \times 10^{5}$ per well for $24 \mathrm{~h}$. Then, the cells were transiently transfected with NCsiRNA $(25 \mathrm{nM})$ or SIRT1-siRNA $(25 \mathrm{nM})$ by Lipofectamine 2000 (Invitrogen) for $6 \mathrm{~h}$, respectively, following the manufacturer's instructions [27]. Then, the media of cells were replaced with MEM (10\% FBS) without penicillin-streptomycin. NC-siRNA (si-NC) and SIRT1-siRNA (si-SIRT1) were obtained from Santa Cruz Biotechnology (Santa Cruz, CA, USA), and the sequences are listed in Table 1.

2.9. Quantitative Real-Time Polymerase Chain Reaction ( $q R T-P C R)$. All primers were synthesized by RiboBio Co. (Guangzhou, China). Total RNA was isolated using TRIzol (Invitrogen, Carlsbad, CA) according to the manufacturer's recommendations. For detection of mRNA, total RNA (2 $\mu \mathrm{g})$ was transcribed into cDNA using AMV Reverse Transcriptase (Promega, Madison, WI), according to the manufacturer's instructions. Primer sequences are shown in Table 2. The qRT-PCR was performed using the LightCycler 96 SYBR Green I Master Mix (Roche).

2.10. Statistical Analysis. Data sets were compared using GraphPad 6.0 (GraphPad Software, Inc., La Jolla, CA, USA). Data are presented as the mean \pm standard deviation (SD). The differences between two groups were analyzed using two-tailed Student's $t$-test. For the repeated measure data, a one- or two-way analysis of variance (ANOVA) followed by a Sidak's multiple comparisons test was used. Differences with $P$ values $<0.05$ were considered statistically significant.

\section{Results}

3.1. Effects of Acetylation on 27HC-Induced Senescence in Neural Cells. Our previous work showed that 27HC can induce STAT3 phosphorylation at Tyr705, and this was involved in nerve cell senescence [5]. Both acetylation and phosphorylation of STAT3 play important roles in transcriptional regulation. In the present research, we investigated whether STAT3 acetylation is also involved in $27 \mathrm{HC}$ induced neural cell senescence. BV2 and PC12 cells were treated with $10 \mu \mathrm{M} 27 \mathrm{HC}, 10 \mu \mathrm{M} 27 \mathrm{HC}+10 \mu \mathrm{M} \mathrm{C} 646$, or $10 \mu \mathrm{M}$ C646 alone for $12 \mathrm{~h}$ and then subjected to an SA- $\beta$ Gal staining assay. We found that $27 \mathrm{HC}$ increased the number of SA- $\beta$-Gal-positive cells. C646, a selective inhibitor of the acetyltransferase P300 associated with STAT3 acetylation, significantly reduced the number of SA- $\beta$-Gal-positive cells increased by $27 \mathrm{HC}$ (Figures $1(\mathrm{a})$ and $1(\mathrm{~b})$ ), indicating that acetylation may be involved in 27HC-induced senescence in neural cells.

3.2. 27HC Induces STAT3 Activation via SIRT1. SIRT1 is an NAD-dependent deacetylase that is located in the nucleus. We explored whether SIRT1 can deacetylate STAT3 at Lys685. We initially treated BV2 and PC12 cells with $10 \mu \mathrm{M}$ $27 \mathrm{HC}$ or $10 \mathrm{nM} \mathrm{E}_{2}$. We observed that $27 \mathrm{HC}$ and $\mathrm{E}_{2}$ increased 
TABLE 1: siRNAs used in this study.

\begin{tabular}{|c|c|c|c|}
\hline Names & Web link & Source & Used \\
\hline $\begin{array}{l}\text { SIRT1 siRNA } \\
\text { (BV2) }\end{array}$ & $\begin{array}{c}\text { https://www.scbt.com/zh/p/sirt1-sirna-m-shrna-and-lentiviral-particle-gene- } \\
\text { silencers }\end{array}$ & $\begin{array}{c}\text { Santa Cruz } \\
\text { Biotechnology }\end{array}$ & $\begin{array}{l}25 \\
\mathrm{nM}\end{array}$ \\
\hline NC siRNA (BV2) & $\begin{array}{c}\text { Sllencers } \\
\text { https://www.scbt.com/zh/p/control-sirna-a }\end{array}$ & $\begin{array}{c}\text { Santa Cruz } \\
\text { Biotechnology }\end{array}$ & $\begin{array}{l}25 \\
\mathrm{nM}\end{array}$ \\
\hline $\begin{array}{l}\text { SIRT1 siRNA } \\
(\mathrm{PC} 12)\end{array}$ & $\begin{array}{c}\text { https://http://www.scbt.com/zh/p/sirt1-sirna-r-shrna-and-lentiviral-particle-gene- } \\
\text { silencers }\end{array}$ & $\begin{array}{l}\text { Santa Cruz } \\
\text { Biotechnology }\end{array}$ & $\begin{array}{l}25 \\
\mathrm{nM}\end{array}$ \\
\hline NC siRNA (PC12) & https://http://www.scbt.com/zh/p/control-sirna-a & $\begin{array}{l}\text { Santa Cruz } \\
\text { Biotechnology }\end{array}$ & $\begin{array}{l}25 \\
\mathrm{nM}\end{array}$ \\
\hline
\end{tabular}

TABle 2: Primer sequence.

\begin{tabular}{|c|c|}
\hline Genes & Primers $\left(5^{\prime}-3^{\prime}\right)$ \\
\hline RAT-IL-6 & $\begin{array}{l}\text { Forward: CAGCCAGTTGCCTTCTTG } \\
\text { Reverse: TGGTCTGTTGTGGGTGGT }\end{array}$ \\
\hline RAT-SIRT1 & $\begin{array}{l}\text { Forward: GTCTGTGCCTTCCAGTTGCT } \\
\text { Reverse: CTGCTTGCTGTCCATACCTG }\end{array}$ \\
\hline RAT- $\beta$-actin & $\begin{array}{c}\text { Forward: AAGTCCCTCACCCTCCCAAAAG } \\
\text { Reverse: AAGCAATGCTGTCACCTTCCC }\end{array}$ \\
\hline Mouse-IL-6 & $\begin{array}{l}\text { Forward: ACCAGAGGAAATTTTCAATAGGC } \\
\text { Reverse: TGATGCACTTGCAGAAAACA }\end{array}$ \\
\hline Mouse-SIRT1 & $\begin{array}{l}\text { Forward: GGAACCTTTGCCTCATCT } \\
\text { Reverse: CTGGAACCAACAGCCTTA }\end{array}$ \\
\hline Mouse- $\beta$-actin & $\begin{array}{l}\text { Forward: CGATGCCCTGAGGCTCTTT } \\
\text { Reverse: TGGATGCCACAGGATTCCAT }\end{array}$ \\
\hline Zebrafish-IL-6 & $\begin{array}{c}\text { Forward: AAGGGGTCAGGATCAGCAC } \\
\text { Reverse: GCTGTAGATTCGCGTTAGACATC }\end{array}$ \\
\hline Zebrafish-SIRT1 & $\begin{array}{l}\text { Forward: CAGCTCTGCTACAATTCATCGCGTC } \\
\text { Reverse: AATCTCTGTAGATCCAGCGCGTGTG }\end{array}$ \\
\hline
\end{tabular}

the expression of Ac-STAT3 ${ }^{\text {Lys685 }}$ but decreased the expression of SIRT1 in BV2 cells. 27HC also increased the expression of Ac-STAT3 ${ }^{\text {Lys685 }}$ and decreased the expression of SIRT1 in PC12 cells, but no significant difference was observed in the effects of E2 on the two proteins compared with CON (Figures 2(a) and 2(b)To investigate the role of SIRT1 in STAT3 phosphorylation and acetylation, siRNASIRT1 was used to knockdown the expression of SIRT1 in BV2 and PC12 cells. We found that siRNA-SIRT1 increased the levels of p-STAT3 $3^{\text {Tyr705 }}$ and Ac-STAT3 ${ }^{\text {Lys685 }}$ in both BV2 and PC12 cells (Figures 2(c) and 2(d)) and also increased the mRNA expression of interleukin 6 (IL-6), a gene related to STAT3 signal activation (Figure 2(e)). Taken together, these data indicated that STAT3 acetylation at Lys685 played a crucial role in 27HC-triggered cellular senescence in neural cells and SIRT1 was involved in 27HC-induced STAT3 activation.

3.3. 27HC Downregulates SIRT1 Expression by ROS-Mediated Methylation. As described above, our data showed that $27 \mathrm{HC}$ can inhibit SIRT1 and increased Ac-STAT3 ${ }^{\text {Lys685. }}$. Studies have shown that ROS induces cell DNA damage as well as DNA methylation leading to the silencing of some genes [28]. In the present study, we explored whether the regula- tion of SIRT1 genes by $27 \mathrm{HC}$ was associated with epigenetic changes. BV2 and PC12 cells were exposed to $10 \mu \mathrm{M} 27 \mathrm{HC}$, $100 \mu \mathrm{M}$ SAM, $10 \mu \mathrm{M} 27 \mathrm{HC}+2 \mathrm{mMNAC}$, and $10 \mu \mathrm{M} 27$ $\mathrm{HC}+2.5 \mu \mathrm{M}$ AZA, respectively. We found that $27 \mathrm{HC}$ or SAM (methyl-donor) alone promoted the expression of the DNA methyltransferase DNMT1 and inhibited the expression of SIRT1. Simultaneously, the addition of the methyltransferase inhibitor AZA reversed the upregulation of DNMT1 and inhibition of SIRT1 induced by $27 \mathrm{HC}$ (Figures 3(a)-3(d)). Moreover, we also observed that the addition of the ROS scavenger NAC reversed the inhibitory effect of 27HC on SIRT1 at both an mRNA (Figures 3(e) and 3(f)) and protein level (Figures 3(a)-3(d)). These findings suggested that 27HC downregulated SIRT1 expression through DNA methylation mediated by ROS.

3.4. REV Alleviates 27HC-Induced Senescence and STAT3 Activation. To confirm whether the antioxidant properties of REV were related to 27HC-induced cellular senescence, we treated BV2 and PC12 cells with $10 \mu \mathrm{M} 27 \mathrm{HC}, 10 \mu \mathrm{M} 27$ $\mathrm{HC}+20 \mu \mathrm{M} \mathrm{REV}$, or $20 \mu \mathrm{M} \mathrm{REV}$ alone for $12 \mathrm{~h}$. Interestingly, REV significantly attenuated the 27HC-enhanced number of SA- $\beta$-Gal-positive cells in both cell types 


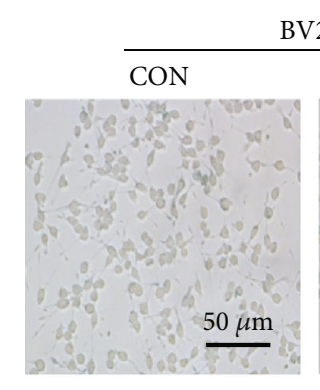

$27 \mathrm{HC}+\mathrm{C} 646$
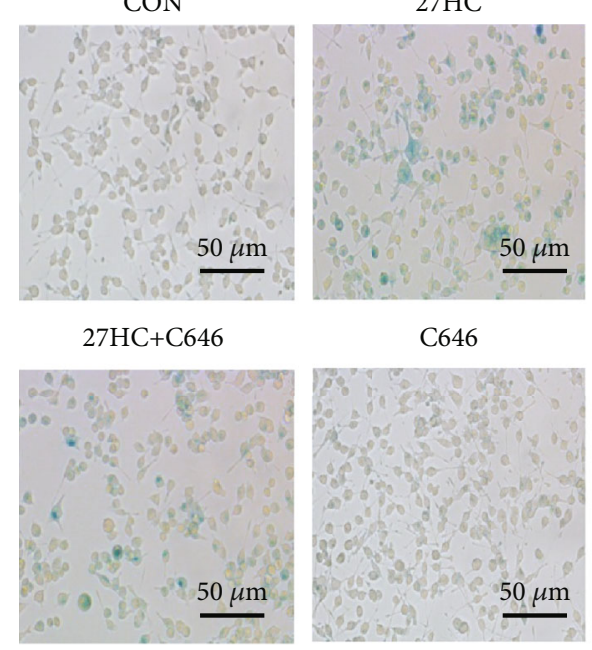

C646

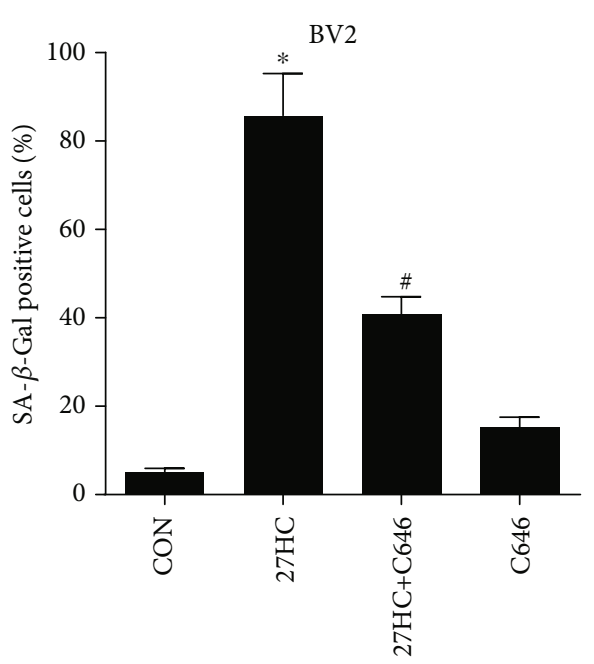

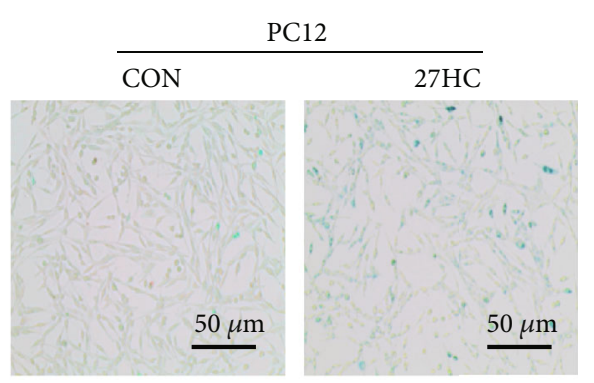

$27 \mathrm{HC}+\mathrm{C} 646$

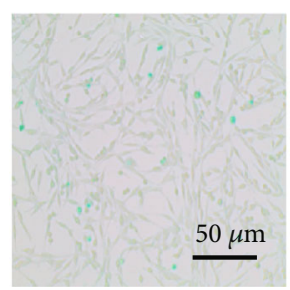

(a)

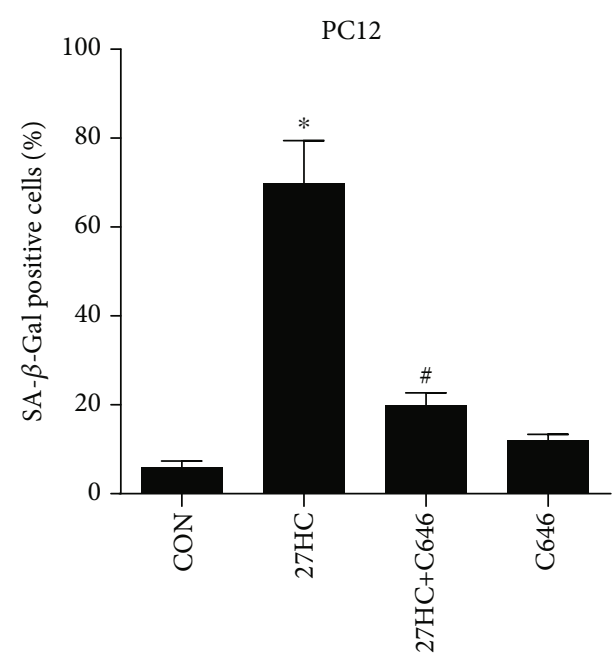

(b)

FIGURE 1: Effects of acetylation on 27HC-induced senescence in neural cells. BV2 and PC12 cells were pretreated with $10 \mu \mathrm{M} 27 \mathrm{HC}$ or $10 \mu \mathrm{M}$ $27 \mathrm{HC}$ plus $10 \mu \mathrm{M}$ C646 for $12 \mathrm{~h}$. CON group: ethanol and DMSO treatment. (a) Cells were subjected to an SA- $\beta$-Gal staining assay. Scale bars: $50 \mu \mathrm{m}$. (b) The aged cells were stained in blue, and the stained cells from panels of BV2 and PC12 cells were counted. ${ }^{*} P<0.05$, statistically significant difference from the CON group (BV2: $P<0.0001$; PC12: $P<0.0001$ ). ${ }^{\#} P<0.05$, statistically significant difference from the 27HC-treated group (BV2: $P=0.0001$; PC12: $P<0.0001$ ). Data are expressed as the mean \pm SD of three independent experiments.

(Figures 4(a) and 4(b)). Both acetylation and phosphorylation of STAT3 play important roles in transcriptional regulation. So, we investigated the effect of REV on STAT3 in neural cells. BV2 and PC12 cells were exposed to $10 \mu \mathrm{M}$ $27 \mathrm{HC}$ or $10 \mu \mathrm{M} 27 \mathrm{HC}+20 \mu \mathrm{M} \mathrm{REV}$ for $12 \mathrm{~h}$. We found that REV weakened the suppressive function of $27 \mathrm{HC}$ on SIRT1 and inhibited the expression of p-STAT3 ${ }^{\text {Tyr705 }}$ and AcSTAT3 ${ }^{\text {Lys } 685}$ induced by $27 \mathrm{HC}$ (Figures $4(\mathrm{c})$ and $4(\mathrm{~d})$ ). In addition, REV also inhibited the 27HC-induced increase in IL-6 mRNA (Figure 4(e)). The above results indicated that REV attenuated 27HC-enhanced cellular senescence and inhibited the activation of STAT3 induced by $27 \mathrm{HC}$.

3.5. Effects of 27HC on ROS Generation in Zebrafish Larva. The zebrafish is inexpensive to maintain and has favorable characteristics for experimentation, such as a high fecundity, rapid external development, embryonic translucence, and ease of genetic manipulation. The senescence process in zebrafish shares similarities with humans and mammals to a certain extent, and zebrafish have been gradually used in the study of aging biology in recent years [29]. Therefore, we used zebrafish as an in vivo experimental model to further verify the effect of $27 \mathrm{HC}$ on neurobehavior and aging. We first observed zebrafish embryo mortality after exposure to $0,10,30$, and $40 \mu \mathrm{M} 27 \mathrm{HC}$ from $36 \mathrm{hpf}$ to $4 \mathrm{dpf}$. The results showed that zebrafish embryo mortality was increased in a dose-dependent manner (Figure 5(a)). Then, we selected $20 \mu \mathrm{M} 27 \mathrm{HC}$ (zebrafish embryo mortality $<5 \%$ ) as the experimental dose. Zebrafish larvae were treated with $20 \mu \mathrm{M} 27 \mathrm{HC}, 10 \mathrm{nM} \mathrm{E}_{2}$, and $20 \mu \mathrm{M} 27 \mathrm{HC}+20 \mu \mathrm{M} \mathrm{NAC}$ 


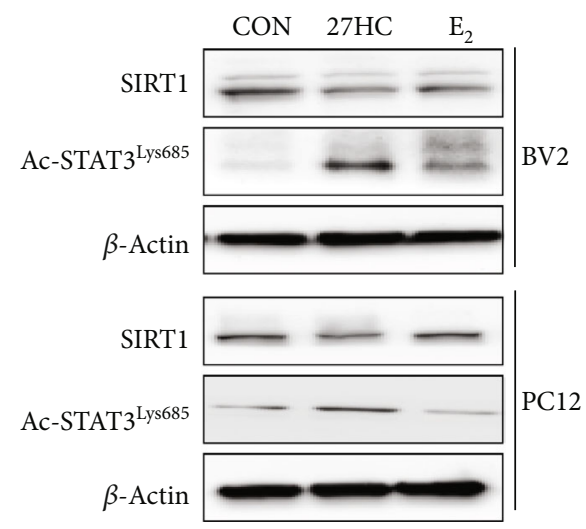

(a)
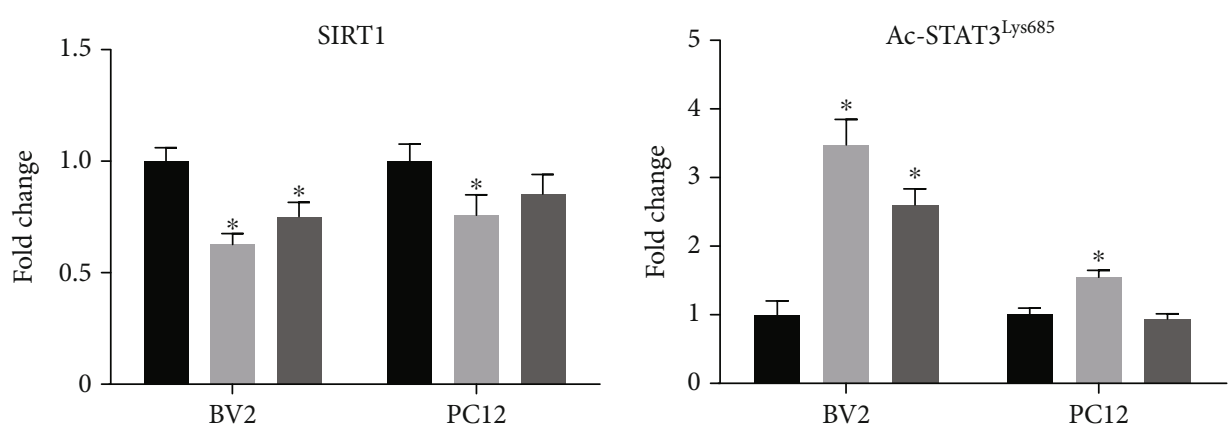

- $\mathrm{CON}$

$27 \mathrm{HC}$

- $\mathrm{E}_{2}$

(b)

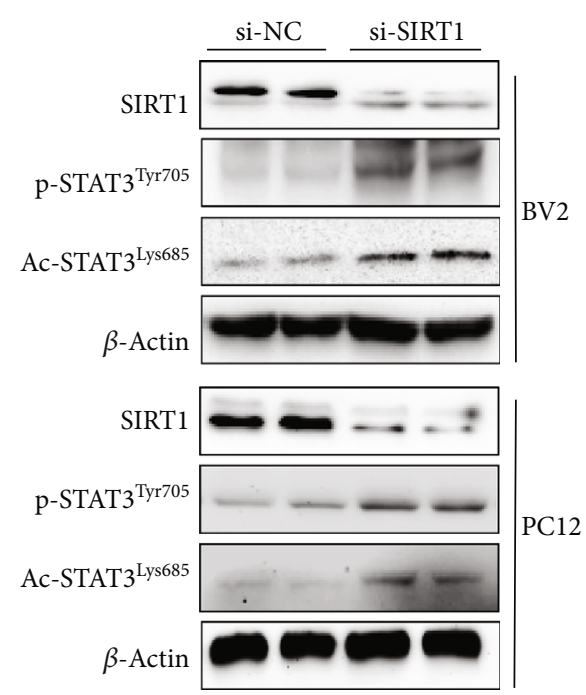

(c)

Figure 2: Continued. 


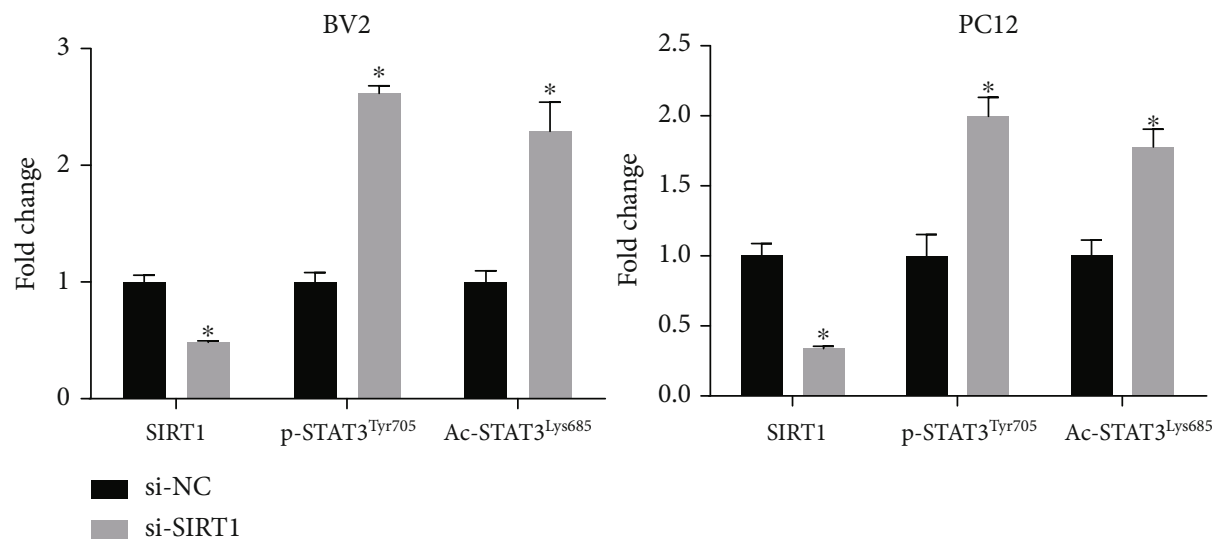

(d)
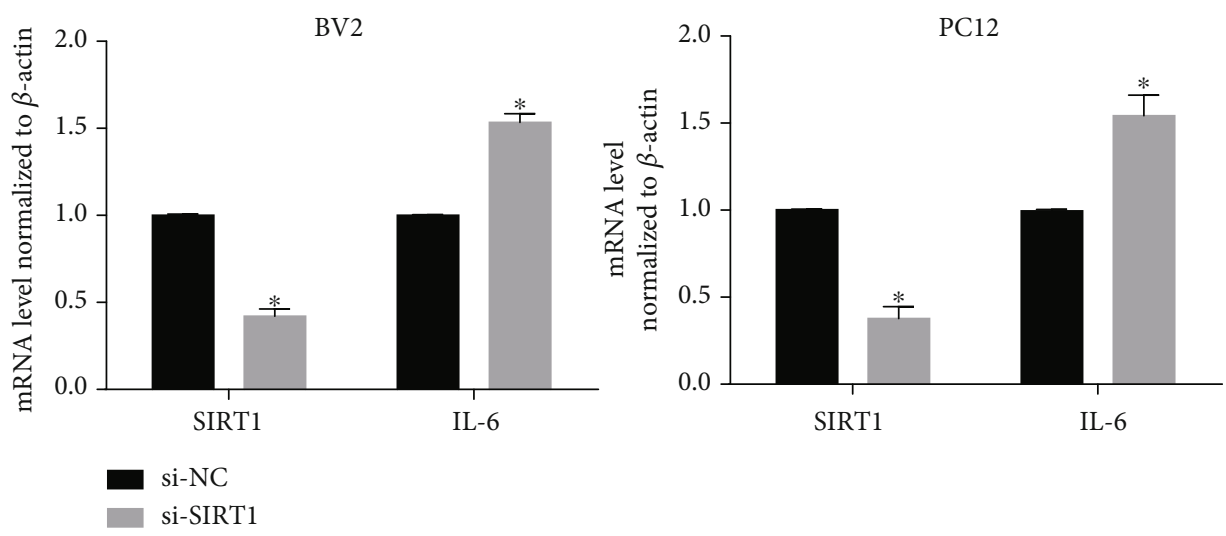

(e)

FIgURE 2: The 27HC induces STAT3 activation via SIRT1. (a) BV2 and PC12 cells were treated with $10 \mu \mathrm{M} 27 \mathrm{HC}$ and $10 \mathrm{nM}$ E2. Western blot analysis was performed for SIRT1, Ac-STAT3Lys685, and $\beta$-Actin expression. CON group: ethanol treatment. (b) The quantitative analysis of protein bands of (a). ${ }^{*} P<0.05$, statistically significant difference from the CON group. SIRT1: (BV2: $P=0.0011,27 \mathrm{HC}$ group; $P=0.0082, \mathrm{E}_{2}$ group) (PC12: $P=0.0248,27 \mathrm{HC}$ group; $P=0.0947, \mathrm{E} 2$ group); AC-STAT3 ${ }^{\text {Lys685 }}$ : (BV2: $P=0.0005,27 \mathrm{HC}$ group; $P=0.0009, \mathrm{E}_{2}$ group) (PC12: $P=0.0026,27 \mathrm{HC}$ group; $P=0.4597, \mathrm{E}_{2}$ group). Data are expressed as the mean $\pm \mathrm{SD}$ of three independent experiments. (c) BV2 and PC12 cells were treated with si-NC or siRNA-SIRT1 for $12 \mathrm{~h}$. Western blot analysis was performed for SIRT1, p-STAT3 ${ }^{\text {Tyr705, Ac- }}$ STAT3 $^{\text {Lys685}}$, and $\beta$-actin expression. The two sets are experimental replicates. (d) The quantitative analysis of protein bands of (c). ${ }^{*} P<0.05$, statistically significant difference from the si-NC group. BV2: $P<0.0001$, SIRT $1 ; P<0.0001$, p-STAT3 ${ }^{\text {Tyr705 }} ; P<0.0001$, Ac-STAT3 ${ }^{\text {Lsy685 }}$; PC12: $P<0.0001$, SIRT1; $P=0.0003$, p-STAT3 ${ }^{\text {Tyr705 }} ; P=0.0001$, Ac-STAT3 ${ }^{\text {Lsy685 }}$. (e) qRT-PCR analysis of IL-6 and SIRT1. ${ }^{*} P<0.05$, statistically significant difference from the si-NC group. (BV2: $P=0.0027$, SIRT1; $P=0.0044$, IL-6; PC12: $P<0.0001$, SIRT1; $P=0.0013$, IL-6). Data are expressed as the mean \pm SD of three independent experiments.

for $36 \mathrm{hpf}$ to $4 \mathrm{dpf}$. In vivo staining for ROS at $4 \mathrm{dpf}$ with DCFH-DA revealed the presence of high levels of ROS in zebrafish larvae exposed to $27 \mathrm{HC}$, which was insignificant in $10 \mathrm{nM} \mathrm{E}_{2}$-exposed larvae. The effect of $27 \mathrm{HC}$ on ROS generation in zebrafish larvae was also inhibited following combination with NAC (Figures 5(b) and 5(c)).

3.6. REV Attenuated 27HC-Induced Aging in Zebrafish Larvae. The zebrafish embryos were exposed to $20 \mu \mathrm{M}$ $27 \mathrm{HC}, 10 \mathrm{nM} \mathrm{E}_{2}, 20 \mu \mathrm{M} 27 \mathrm{HC}+20 \mu \mathrm{MNAC}$, or $20 \mu \mathrm{M} 27$ $\mathrm{HC}+1 \mu \mathrm{M} \mathrm{REV}$ for $36 \mathrm{hpf}$ to $4 \mathrm{dpf}$. Staining for SA- $\beta-\mathrm{Gal}$ in the neural spinal cord was found to increase in the zebrafish embryos following 27HC exposure, yet the staining pattern was distinct from $\mathrm{E}_{2}$. Further, we found that $20 \mu \mathrm{M}$ NAC or $1 \mu \mathrm{M}$ REV could reduce the 27HC-induced SA- $\beta$-Gal activity (Figures 5(d) and 5(e)). These results showed that
REV improved the 27HC-induced aging in the neural spinal cord of zebrafish larvae.

3.7. Effects of REV on 27HC-Induced Disorders in Locomotor Behavior of Zebrafish Larvae. The effects of $27 \mathrm{HC}$ on locomotor behavior and behavioral parameters of zebrafish larva were evaluated by EthoVision XT software Noldus IT after a $36 \mathrm{hpf}$ to $4 \mathrm{dpf}$ exposure to $20 \mu \mathrm{M} 27 \mathrm{HC}, 10 \mathrm{nM} \mathrm{E}_{2}, 20 \mu \mathrm{M}$ $27 \mathrm{HC}+20 \mu \mathrm{M} \mathrm{NAC}$, or $20 \mu \mathrm{M} 27 \mathrm{HC}+1 \mu \mathrm{M} \mathrm{REV}$. We visualized the locomotor trajectories of the $4 \mathrm{dpf}$ larvae in the dark for $3 \mathrm{~min}$. The results showed that $70 \%$ of the larvae in the 27HC-exposed group no longer turned routinely along the inner wall of the 96-well plate compared to the control group, which was accompanied by an increase in spontaneous locomotor trajectories, stereotyped steering, small-scale circle phenomena, and touch-wall reflex activity reduction. 


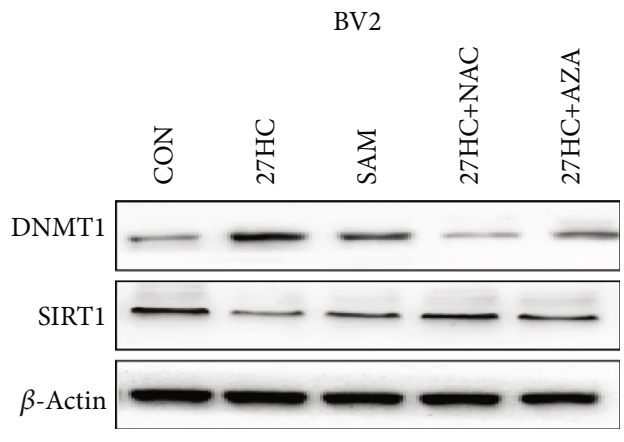

(a)
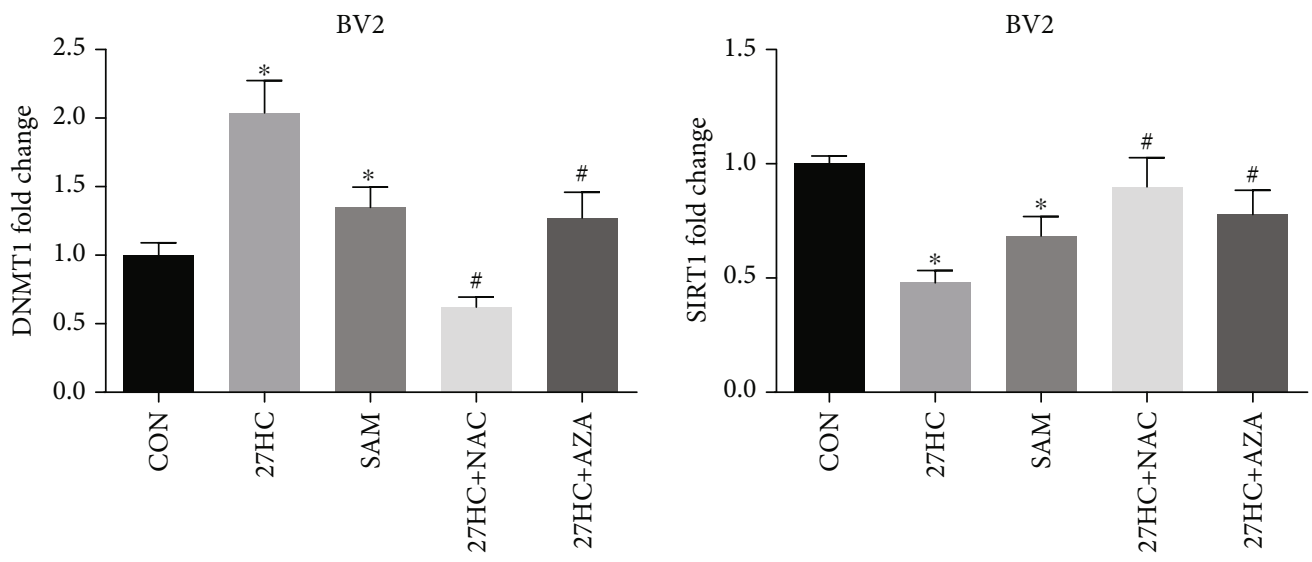

(b)

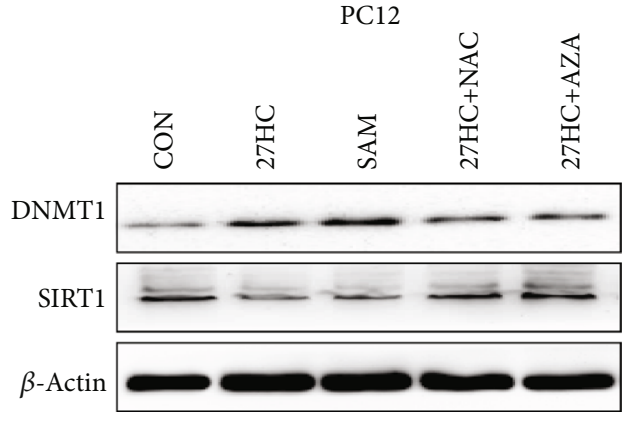

(c)
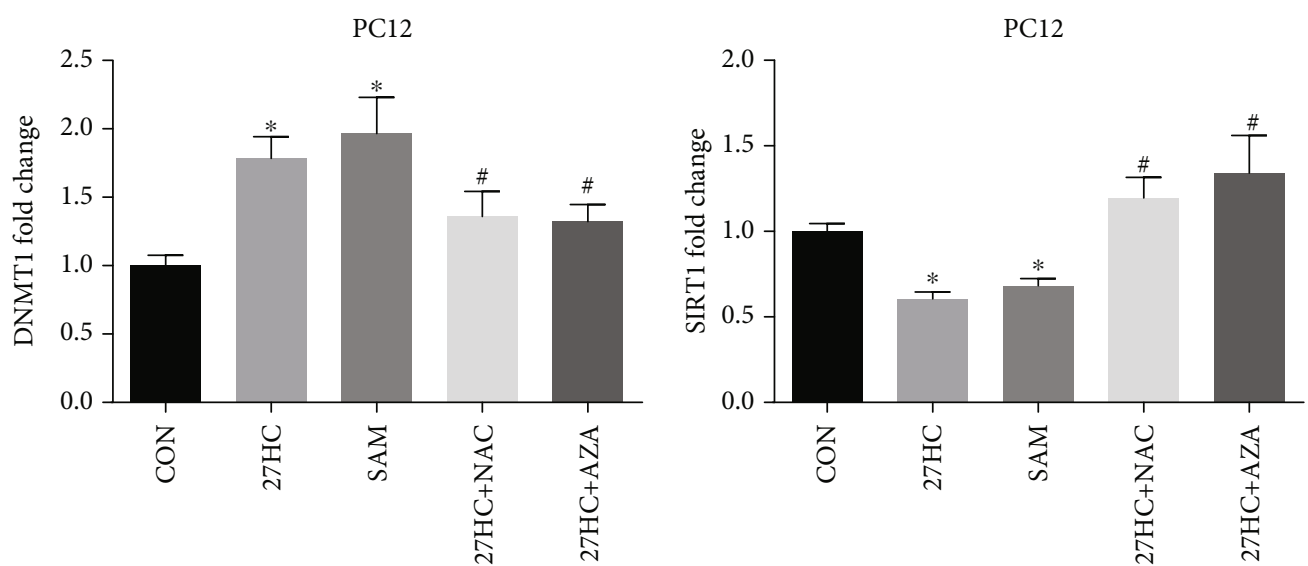

(d)

Figure 3: Continued. 


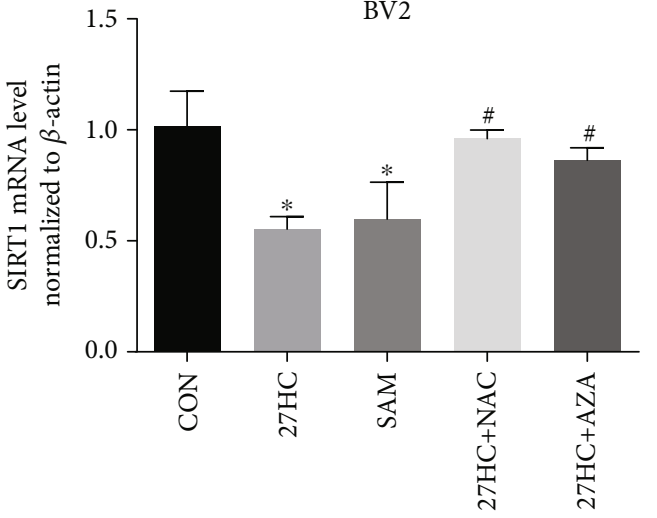

(e)

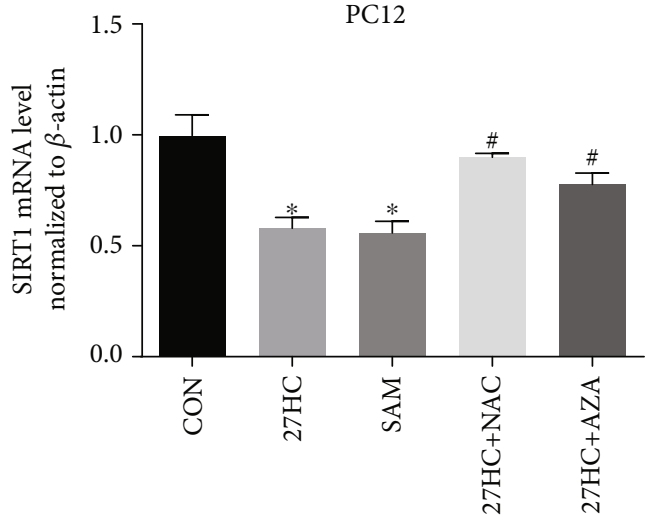

(f)

Figure 3: The 27HC downregulates SIRT1 expression via ROS-mediated methylation. BV2 and PC12 cells were treated with $10 \mu \mathrm{M} 27 \mathrm{HC}$, $100 \mu \mathrm{M}$ SAM, $10 \mu \mathrm{M} 27 \mathrm{HC}+2 \mathrm{mM}$ NAC (pretreatment for $2 \mathrm{~h}$ ), and $10 \mu \mathrm{M} 27 \mathrm{HC}+2.5 \mu \mathrm{M}$ AZA (pretreatment for $2 \mathrm{~h}$ ) for $12 \mathrm{~h}$; CON group: ethanol treatment. ( $\mathrm{a}, \mathrm{c}$ ) Western blot analysis of DNMT1, SIRT1, and $\beta$-actin. (b, d) The quantitative analysis of protein bands of $(\mathrm{a}, \mathrm{c}) . * P<0.05$, statistically significant difference from the CON group. (b) BV2: (DNMT1: $P=0.0020,27 \mathrm{HC}$ group; $P=0.0246$, SAM group; SIRT1: $P=0.0001,27 \mathrm{HC}$ group; $P=0.0039$, SAM group). (d) PC12: (DNMT1: $P=0.0015,27 \mathrm{HC}$ group; $P=0.0036$, SAM group; SIRT1: $P=0.0004,27 \mathrm{HC}$ group; $P=0.0009$, SAM group). ${ }^{\#} P<0.05$, statistically significant difference from the $27 \mathrm{HC}$-treated group. (b) BV2: (DNMT1: $P=0.0006,27 \mathrm{HC}+\mathrm{NAC}$ group; $P=0.0116,27 \mathrm{HC}+\mathrm{AZA}$ group; SIRT1: $P=0.0061,27 \mathrm{HC}+\mathrm{NAC}$ group; $P=0.0124,27 \mathrm{HC}$ +AZA group). (d) PC12: (DNMT1: $P=0.0387,27 \mathrm{HC}+\mathrm{NAC}$ group; $P=0.0166,27 \mathrm{HC}+\mathrm{AZA}$ group; SIRT1: $P=0.0013,27 \mathrm{HC}+\mathrm{NAC}$ group; $P=0.0048,27 \mathrm{HC}+\mathrm{AZA}$ group). (e, f) qRT-PCR analysis of SIRT1. ${ }^{*} P<0.05$, statistically significant difference from the CON group. (e) BV2: $P=0.0014,27 \mathrm{HC}$ group; $P=0.0104$, SAM group. (f) $\mathrm{PC} 12: P=0.0002,27 \mathrm{HC}$ group; $P=0.0002, \mathrm{SAM}$ group. ${ }^{\#} P<0.05$, statistically significant difference from the 27HC-treated group. (e) BV2: $P<0.0001,27 \mathrm{HC}+\mathrm{NAC}$ group; $P=0.0003,27 \mathrm{HC}+\mathrm{AZA}$ group. (f) PC12: $P<0.0001,27 \mathrm{HC}+\mathrm{NAC}$ group; $P=0.0014,27 \mathrm{HC}+\mathrm{AZA}$ group. Data are expressed as the mean $\pm \mathrm{SD}$ of three independent experiments.

At the same time, this irregular movement of larvae was alleviated by combining $27 \mathrm{HC}$ with NAC or REV (Figure 6(a)).

In addition, the analysis of behavioral parameters revealed that, compared with the control group, the larvae moved a shorter distance and had a slower mean speed after $27 \mathrm{HC}$ exposure. The inhibitory effects of $27 \mathrm{HC}$ on the spontaneous movement distance and mean speed of the larvae were weakened following combination with NAC or REV (Figures 6(b) and 6(c)).

The mRNA expression of SIRT1 and IL- 6 in each group was detected by qRT-PCR. The results showed that SIRT1 expression was significantly inhibited after exposure to $27 \mathrm{HC}$, whereas IL-6 expression was increased. After combining $27 \mathrm{HC}$ with NAC or REV, the effects of $27 \mathrm{HC}$ on SIRT1 and IL-6 were reversed (Figures 6(d) and 6(e)). These results were consistent with our in vitro experiments. In the $\mathrm{E}_{2}$ treated group, there was no detectable change in larvae (Figures 6(a)-6(e)). All of the above results showed that 27HC induced locomotor behavior disorders and inflammatory cytokines in zebrafish larvae, and REV alleviated 27HCinduced aging and locomotor behavior disorders.

\section{Discussion}

Animal studies have shown that the passage of serum 27HC through the $\mathrm{BBB}$ can result in the deposition of oxidized cholesterol in the brain, resulting in metabolic disorders of $\beta$-amyloid $(\mathrm{A} \beta)$ and impaired spatial learning and memory [30]. Another study has suggested that high levels of circulating cholesterol increase the entry of $27 \mathrm{HC}$ into the brain, which may induce learning and memory impairment [31]. Based on this evidence, $27 \mathrm{HC}$ may be associated with neurodegenerative processes in the brain. $27 \mathrm{HC}$ may negatively modulate cognitive effects and interrupt neuronal cells and thereby lead to neurodegenerative diseases, such as aging or Alzheimer's disease (AD).

More and more studies have confirmed that SIRT1 plays an important role in the development of neurodegenerative diseases, such as AD, Parkinson's disease (PD), or ischemic stroke [32-34]. It is well-known that the activity of SIRT1 is affected by $\mathrm{NAD}+$, which is an important enzyme substrate for SIRT1. NAD+ is also an electron transporter in the mitochondrial respiratory chain and is affected by the redox state. Therefore, it is believed that the in vivo environment, oxidative stress, and metabolic changes can all affect the acetylation enzyme activity of SIRT1 [35]. More evidence showed that elevated ROS levels can directly or indirectly control SIRT1 enzyme activity. For example, with aging and some age-related diseases, the overproduction of ROS weakens the function of SIRT1 [36]. In addition, ROS can inhibit SIRT1 mRNA levels by inducing microRNA expression [37].

In the present study, we explored whether the regulation of SIRT1 genes by $27 \mathrm{HC}$ was associated with epigenetic changes. We used the methyl-donor SAM and the methyltransferase inhibitor AZA. And the results suggested that 27HC and SAM could promote the DNMT1 expression and inhibit the SIRT1 expression, and the addition of the methyltransferase inhibitor AZA reversed the upregulation of DNMT1 and inhibition of SIRT1 induced by 27HC. Furthermore, the ROS scavenger NAC reversed the effect of 

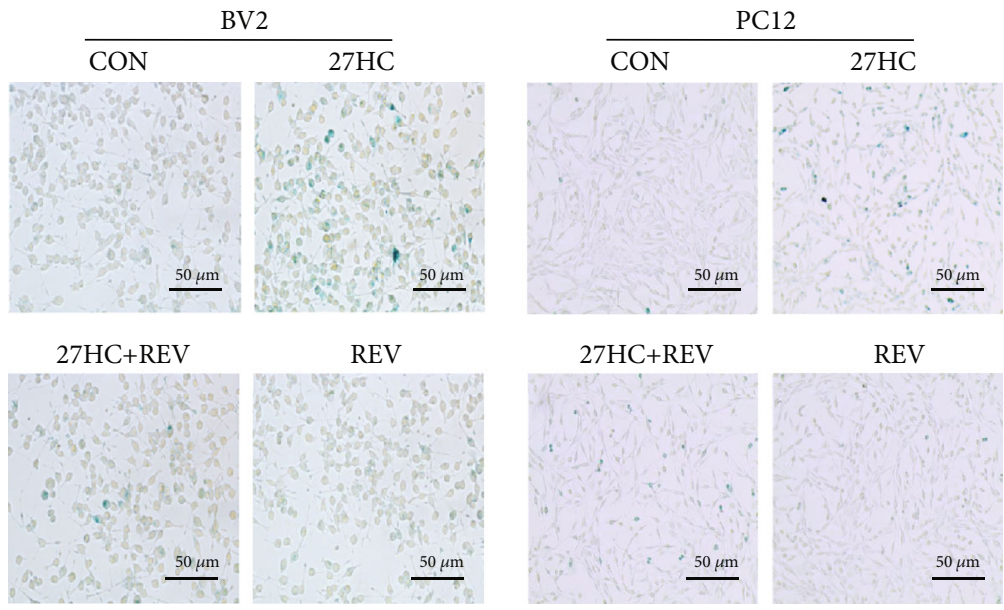

REV

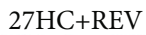

REV
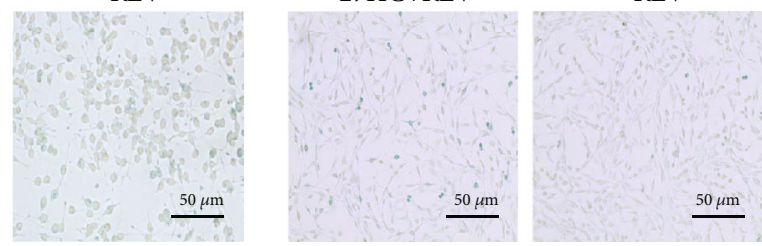

(a)
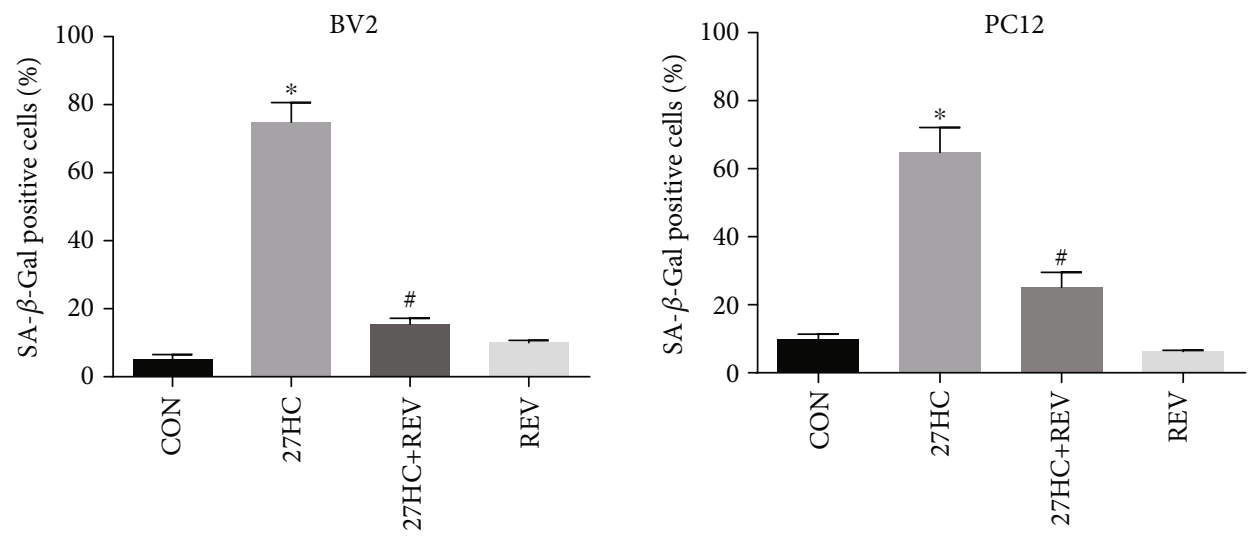

(b)

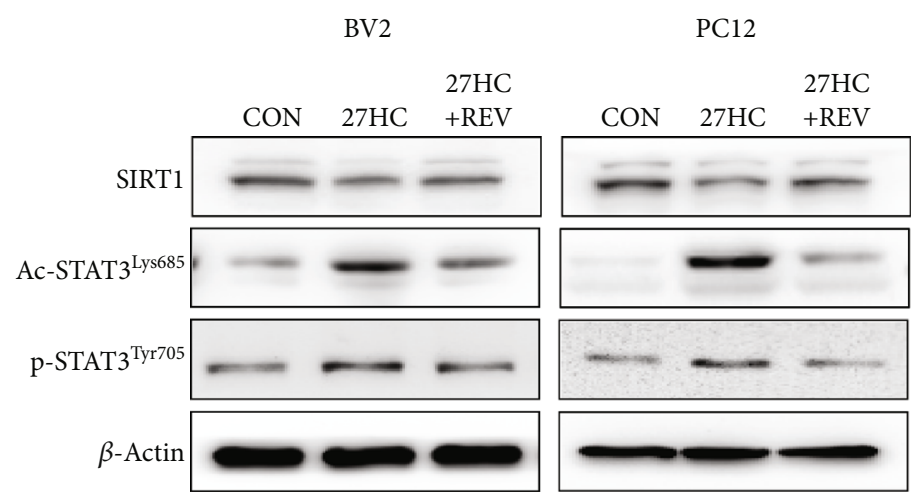

(c)

Figure 4: Continued. 


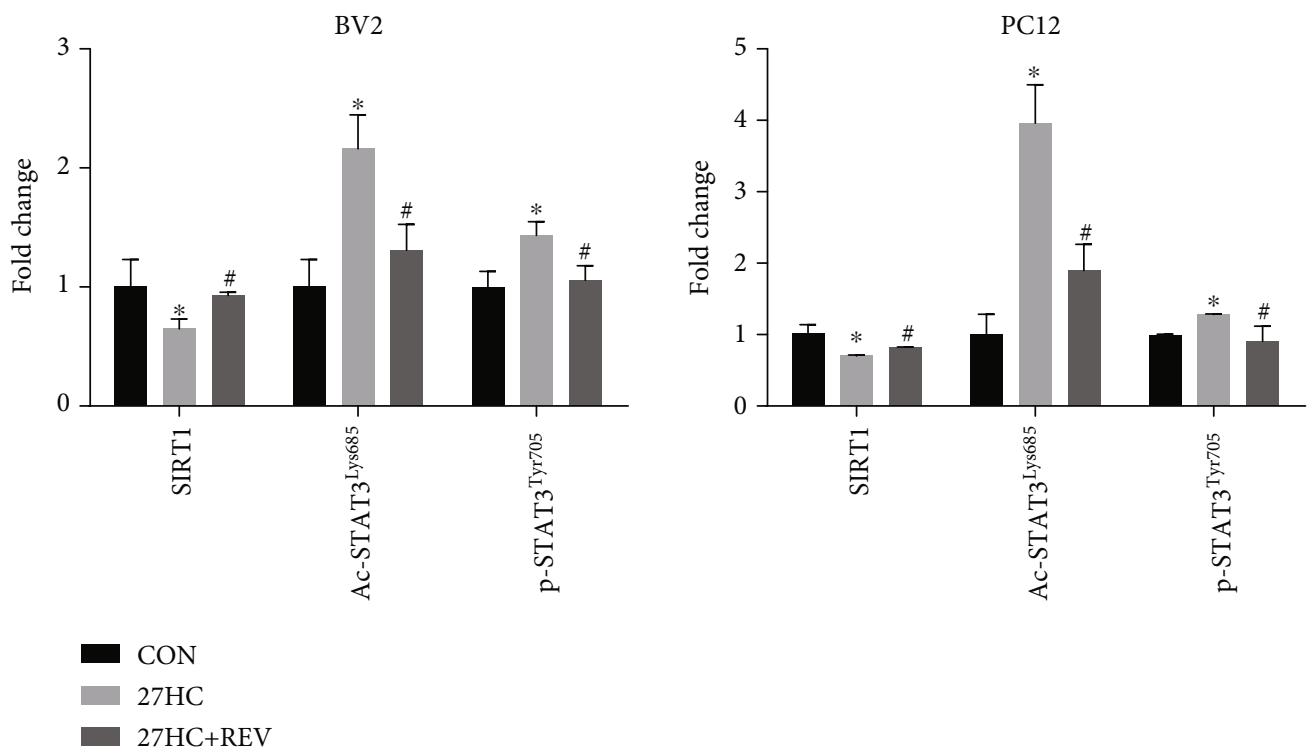

(d)

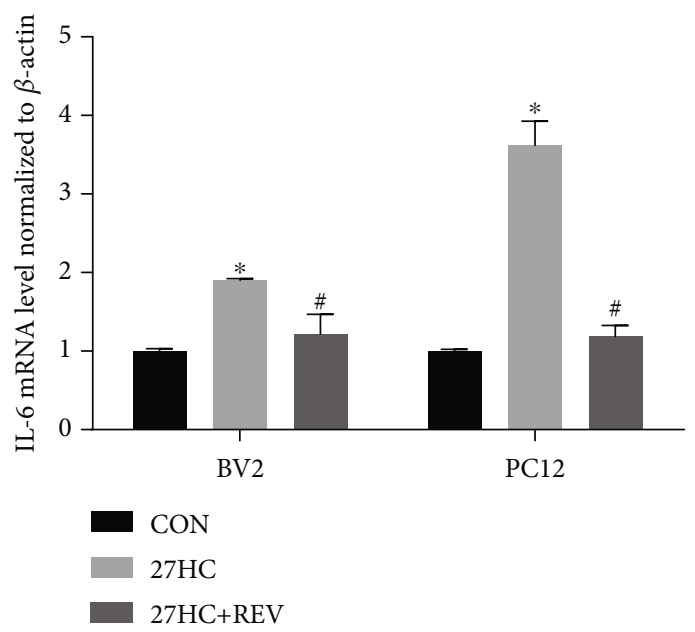

(e)

FIGURE 4: REV inhibits 27HC-induced senescence and STAT3 activation. BV2 and PC12 cells were treated with $10 \mu \mathrm{M} 27 \mathrm{HC}, 10 \mu \mathrm{M} 27$ $\mathrm{HC}+20 \mu \mathrm{M} \mathrm{REV}$, or $20 \mu \mathrm{M} \mathrm{REV}$ alone for $12 \mathrm{~h}$. CON group: ethanol treatment. (a) Cells were subjected to an SA- $\beta$-Gal staining assay. Scale bars: $50 \mu \mathrm{m}$. (b) The aged cells were stained in blue, and the number of stained cells from panels was counted. ${ }^{*} P<0.05$, statistically significant difference from the CON group. BV2: $P<0.0001$; $P C 12: P<0.0001$. ${ }^{*} P<0.05$, statistically significant difference from the 27HC-treated group. BV2: $P<0.0001$; PC12: $P<0.0001$. Data are expressed as the mean \pm SD of three independent experiments. BV2 and PC12 cells were treated with $10 \mu \mathrm{M} 27 \mathrm{HC}$ or $10 \mu \mathrm{M} 27 \mathrm{HC}+20 \mu \mathrm{M} \mathrm{REV}$ for $12 \mathrm{~h}$. CON group: ethanol treatment. (c) Western blot analysis of SIRT1, Ac-STAT3 ${ }^{\text {Lys685 }}$, p-STAT3 ${ }^{\text {Tyr705}}$, and $\beta$-actin. (d) The quantitative analysis of protein bands of $(c)$. ${ }^{*} P<0.05$, statistically significant difference from the CON group. BV2: (SIRT1: $P=0.0224$; Ac-STAT3 ${ }^{\text {Lys685 }}: P=0.0053 ; \mathrm{p}^{-S T A T 3}{ }^{\text {Tyr705}}: P=0.0129$ ); PC12: (SIRT1: $P=0.0155$; Ac-STAT3 ${ }^{\text {Lys685}}: P=0.0011$; $p$-STAT3 ${ }^{\text {Tyr705 }}: P<0.0001$ ). ${ }^{*} P<0.05$, statistically significant difference from the 27HC-treated group. BV2: (SIRT1: $P=0.0007$; Ac-STAT3 ${ }^{\text {Lys685 }}: P=0.0144 ;$ p-STAT3 ${ }^{\text {Tyr705 }}: P=0.0176$ ); PC12: $($ SIRT1: $P<0.0001 ;$ AcSTAT3 ${ }^{\text {Lys685}}: P=0.0053$; p-STAT3 ${ }^{\text {Tyr705 }}: P=0.0368$ ). (e) qRT-PCR analysis of IL- $6 .{ }^{*} P<0.05$, statistically significant difference from the CON group. BV2: $P=0.0006$; PC12: $P<0.0001$. ${ }^{\#} P<0.05$, statistically significant difference from the 27HC-treated group. BV2: $P=0.0023$; PC12: $P<0.0001$. Data are expressed as the mean \pm SD of three independent experiments.

27HC on DNMT1 and SIRT1. Based on the above results, we considered that $27 \mathrm{HC}$ could induce SIRT1 DNA methylation by promoting methyltransferase DNMT1 expression. NNMT may regulate not only SIRT1 gene; whether there are aging-related genes regulated by NNMT in the process of $27 \mathrm{HC}$ induced-aging remains to be further explored. We found that the mRNA level and protein level showed consis- tent changes, and there is no evidence that SIRT1 protein degradation was involved in the process of $27 \mathrm{HC}$-induced cellular senescence, which needs to be further explored. We examined the effects of 27HC and SAM on DNMT1. SAM is a donor which provides the methyl groups for histone or nucleic acid modification. DNMT1 is a key enzyme for DNA replication and repair and maintenance of methylation 

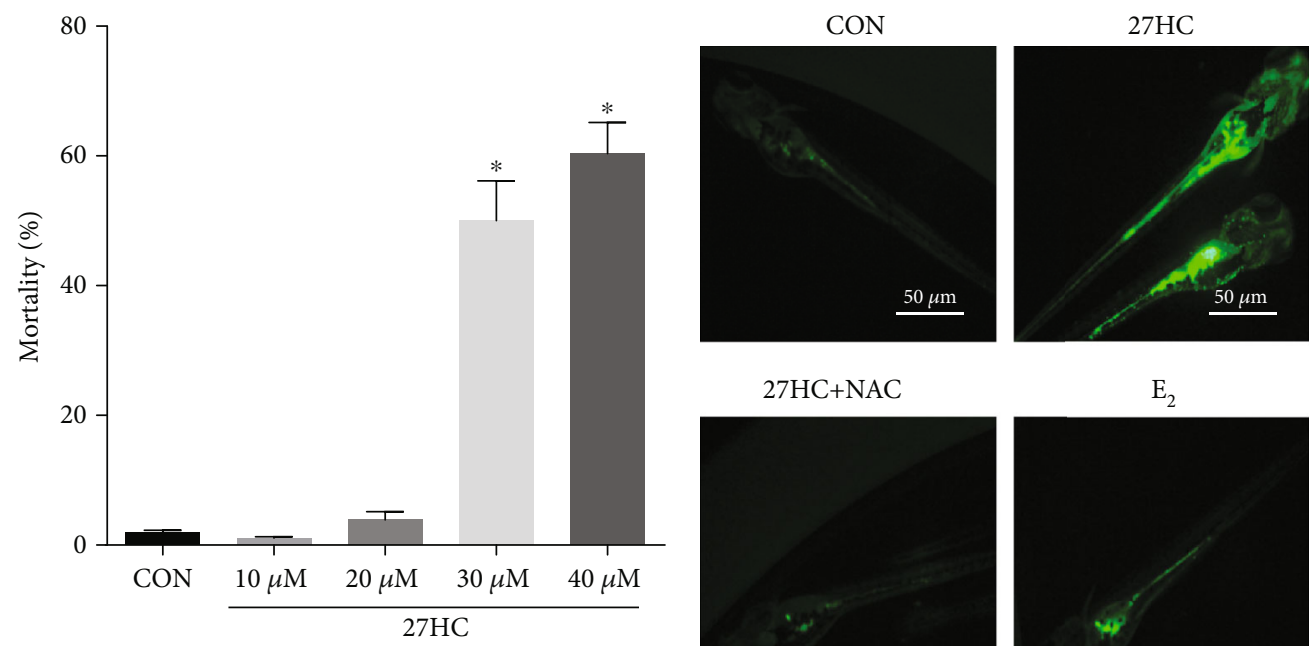

27HC+NAC

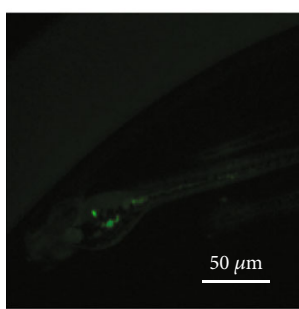

(b)

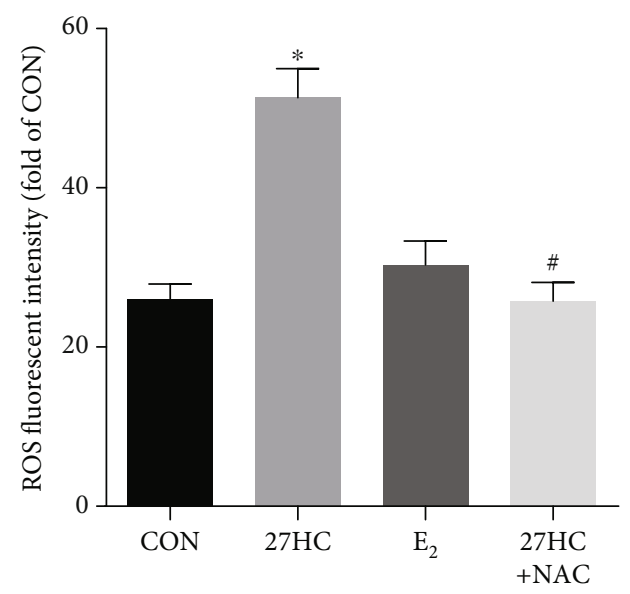

(c)

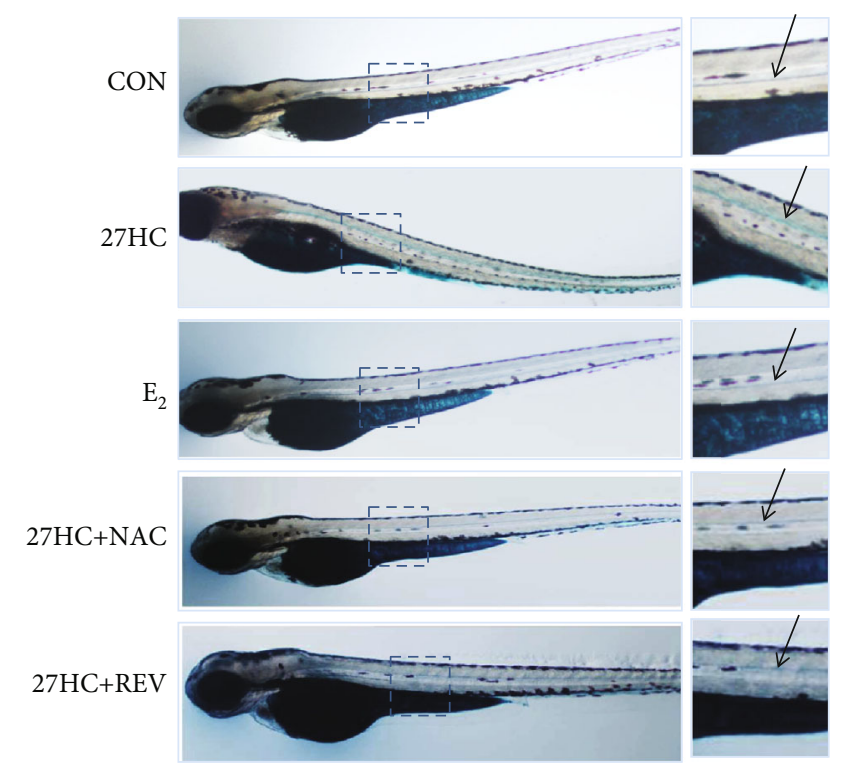

(d)

FIGURE 5: Continued. 


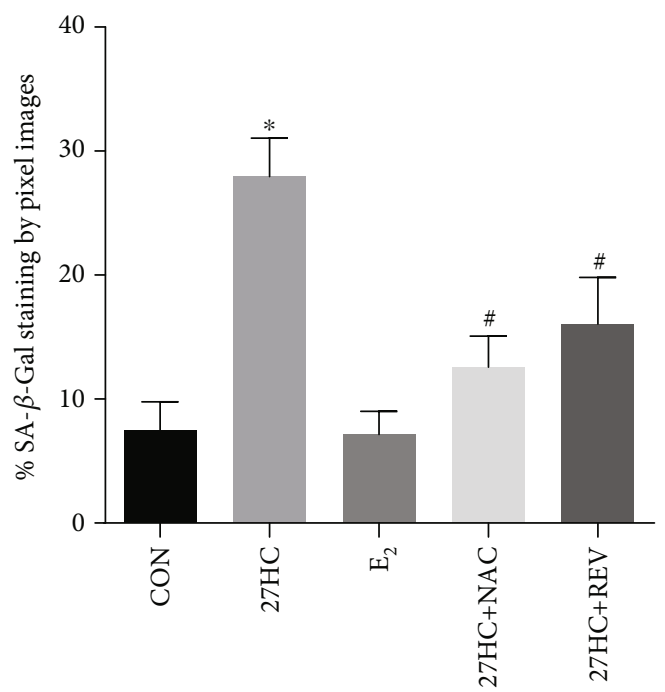

(e)

FiguRE 5: Effects of 27HC on ROS generation in zebrafish larvae. (a) Effect of different concentrations of 27HC on zebrafish embryo mortality $(n=50)$. CON group: ethanol treatment. ${ }^{*} P<0.05$, statistically significant difference from the CON group. $P=0.9927,10 \mu \mathrm{M} 27 \mathrm{HC}$ group; $P=0.9071,20 \mu \mathrm{M}$ 27HC group; $P<0.0001,30 \mu \mathrm{M} 27 \mathrm{HC}$ group; $P<0.0001,40 \mu \mathrm{M} 27 \mathrm{HC}$ group. (b) Staining for ROS in larvae at 4 dpf following $36 \mathrm{hpf}$ to $4 \mathrm{dpf}$ treatment with $20 \mu \mathrm{M} 27 \mathrm{HC}, 20 \mu \mathrm{M} 27 \mathrm{HC}+20 \mu \mathrm{MNAC}$, or $10 \mathrm{nM}$ E2. CON group: ethanol treatment. Scale bars: $50 \mu \mathrm{m}$. (c) The levels of ROS fluorescence were determined using flow cytometric analysis $(n=30)$. REV attenuated 27HC-induced aging in zebrafish larvae. ${ }^{*} P<0.05$, statistically significant difference from the CON group $(P<0.0001)$. ${ }^{\#} P<0.05$, statistically significant difference from the 27HC-treated group $(P<0.0001)$. (d) SA- $\beta$-Gal staining in zebrafish embryos at $4 \mathrm{dpf}$ following $36 \mathrm{hpf}$ to $4 \mathrm{dpf}$ treatment with $20 \mu \mathrm{M} 27 \mathrm{HC}, 10 \mathrm{nM} \mathrm{E} 2,20 \mu \mathrm{M} 27 \mathrm{HC}+20 \mu \mathrm{MNAC}$, or $20 \mu \mathrm{M} 27 \mathrm{HC}+1 \mu \mathrm{M} \mathrm{REV}$. CON group: ethanol treatment. (e) Quantitative analysis of SA- $\beta$-Gal staining in larvae $(n=30) .{ }^{*} P<0.05$, statistically significant difference from the CON group $(P<0.0001)$. ${ }^{\#} P<0.05$, statistically significant difference from the 27HC-treated group. $P=0.0002,27 \mathrm{HC}+\mathrm{NAC}$ group; $P=0.0013,27 \mathrm{HC}+\mathrm{REV}$ group. Data are expressed as mean \pm SD of three independent experiments.

level [38]. So we concluded that the downregulation of SIRT1 was related to upregulation of DNMT1 induced by $27 \mathrm{HC}$. The SAM treatment indirectly shows the expression of DNMT1 and its effect on SIRT1 expression silence, in which as a limitation in the present study, a more conclusive experiment would be to perform bisulfite conversion sequencing or digestion-based PCR assay to demonstrate the DNA methylation site of SIRT1 gene changed by $27 \mathrm{HC}$ in the process of cellular senescence.

The phytochemical REV is a classical SIRT1 agonist, and the covalent binding of REV to SIRT1 alters the molecular conformation of SIRT1 and increases the affinity of the protein for its substrate $[39,40]$. Our study also confirmed that REV can alleviate 27HC-enhanced cellular senescence and the inhibition of SIRT1 induced by $27 \mathrm{HC}$ in neural cells. Our in vivo study further indicated that REV alleviated 27HC-induced spinal cord senescence and locomotor disorders in zebrafish larvae. Extensive research has shown the interventional effects of REV in vitro and vivo, and the form of REV used mostly is prototype rather than its metabolites. In the present study, we also observed that REV prototype alleviated 27HC-induced aging and locomotor behavior disorders in zebrafish. Whether the intervention of REV is its prototype or its metabolites is unclear in this study. Resveratrol has a high metabolism, leading to the production of conjugated sulfates, glucuronides, which retain some biological activity [41]. The fact that resveratrol metabolite was less tested also makes these results poorly translatable. Here, which metabolites of resveratrol are involved in the intervention has not been explored. So, when these questions are clearly identified, we will further explore the intervention functions of resveratrol and its metabolites at physiological concentrations.

Changes in the levels of STAT3 phosphorylation and acetylation were observed in $\mathrm{A} \beta$-induced microglial cell activation models [42]. Therefore, activation of STAT3 also plays an important role in nerve injury and neurodegenerative diseases. Moreover, it is worth noting that the acetylation site of STAT3 is very important for the stability of its dimer formation and gene transcription. Yuan and his colleagues first reported the acetylation of STAT3 at the lysine 685 (Lys685) site, which in turn regulates its dimer formation and DNA-binding capacity [13]. In our previous study, we used a Stattic $(10 \mu \mathrm{M})$ to inhibit the level of p-STAT3, which further inhibited the mRNA expression and secretion of IL6 promoted by $27 \mathrm{HC}$ and $27 \mathrm{HC}$-induced cellular senescence [5], suggesting that STAT3 activation is closely related to 27HC-induced cell senescence. Here, in addition to C646, we also used REV, a naturally occurring phytoalexin that inhibits STAT3 acetylation, and this function is the same with C646 [43]. We found that REV inhibited the expression of p-STAT3 ${ }^{\text {Tyr705 }}$ and Ac-STAT3 ${ }^{\text {Lys685 }}$ promoted by $27 \mathrm{HC}$, which alleviates the 27HC-induced cellular senescence (Figures 4). We considered that STAT3 acetylation may be involved in the cellular senescence induced by $27 \mathrm{HC}$ based on our results. 


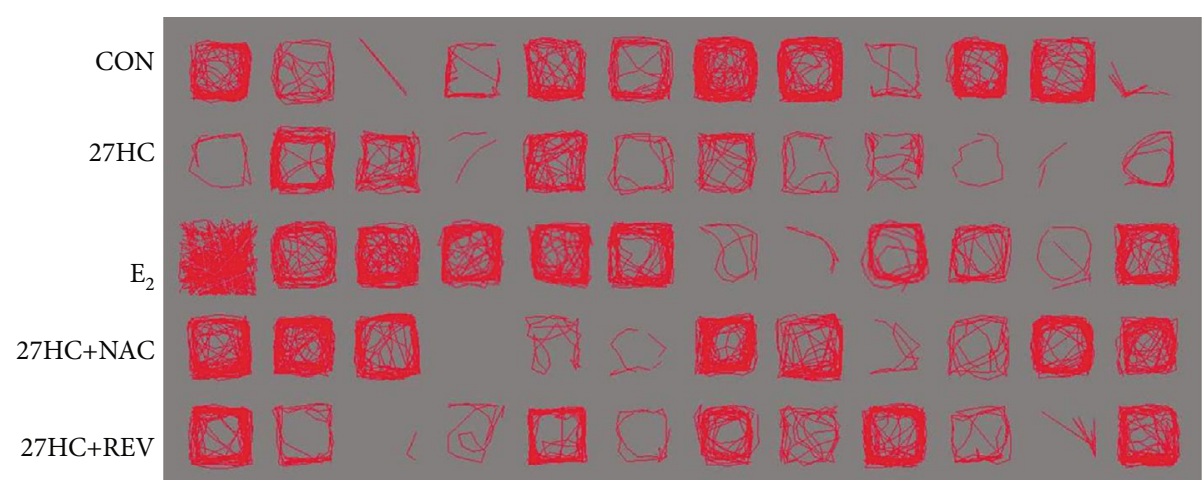

(a)

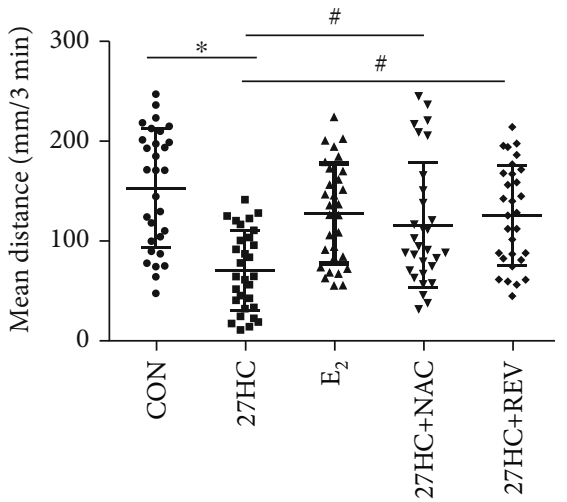

(b)

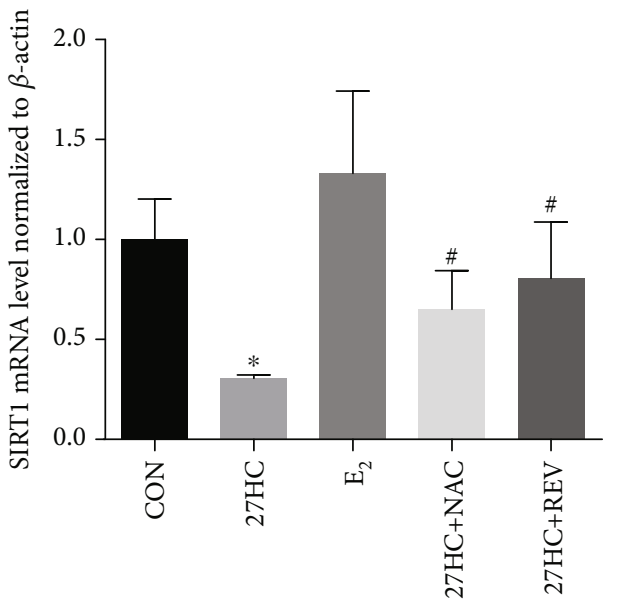

(d)

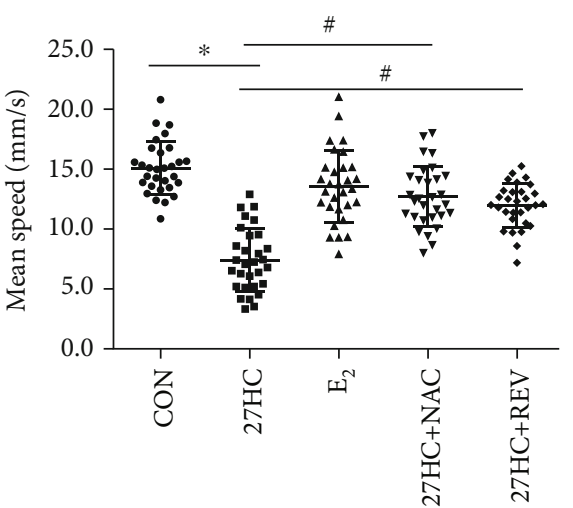

(c)

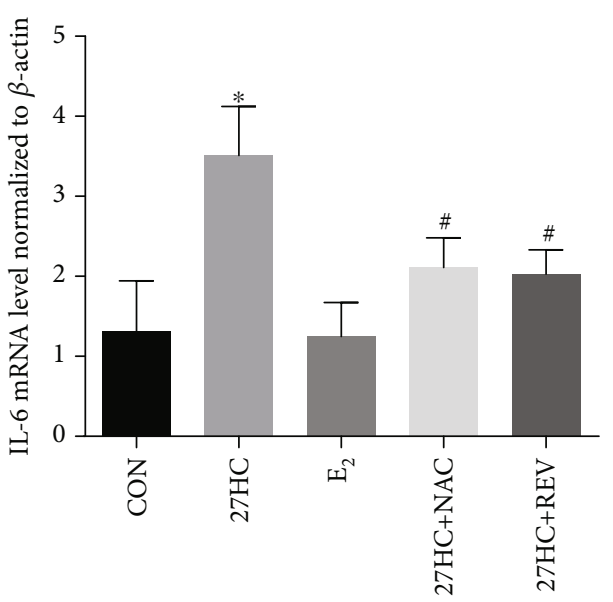

(e)

Figure 6: Effects of REV on 27HC-induced locomotor disorders in zebrafish larvae. Zebrafish larvae from $36 \mathrm{hpf}$ to 4 dpf were exposed to 20 $\mu \mathrm{M} 27 \mathrm{HC}, 10 \mathrm{nM} \mathrm{E} 2,20 \mu \mathrm{M} 27 \mathrm{HC}+20 \mu \mathrm{M} \mathrm{NAC}$, or $20 \mu \mathrm{M} 27 \mathrm{HC}+1 \mu \mathrm{M} \mathrm{REV}$. CON group: ethanol treatment. (a) Locomotor behavior of zebrafish larvae was observed using EthoVision XT software Noldus IT. (b, c) Analysis of behavioral parameters of zebrafish larvae $(n=30)$. ${ }^{*} P<0.05$, statistically significant difference from the CON group. (b) $P<0.0001$. (c) $P<0.0001$. ${ }^{\#} P<0.05$, statistically significant difference from the 27HC-treated group. (b) $P=0.0013,27 \mathrm{HC}+\mathrm{NAC}$ group; $P<0.0001,27 \mathrm{HC}+\mathrm{REV}$ group. (c) $P<0.0001,27 \mathrm{HC}+\mathrm{NAC}$ group; $P<0.0001,27 \mathrm{HC}+\mathrm{REV}$ group. Data are expressed as the mean $\pm \mathrm{SD}$ of three independent experiments. (d, e) qRT-PCR analysis of SIRT1 and IL-6. ${ }^{*} P<0.05$, statistically significant difference from the CON group $(n=30)$. (d) $P=0.0248$. (e) $P=0.0123$. ${ }^{*} P<0.05$, statistically significant difference from the 27HC-treated group. (d) $P=0.0368,27 \mathrm{HC}+\mathrm{NAC}$ group; $P=0.378,27 \mathrm{HC}+\mathrm{REV}$ group. (e) $P=0.0276,27 \mathrm{HC}+\mathrm{NAC}$ group; $P=0.0198,27 \mathrm{HC}+\mathrm{REV}$ group. Data are expressed as the mean $\pm \mathrm{SD}$ of three independent experiments. 


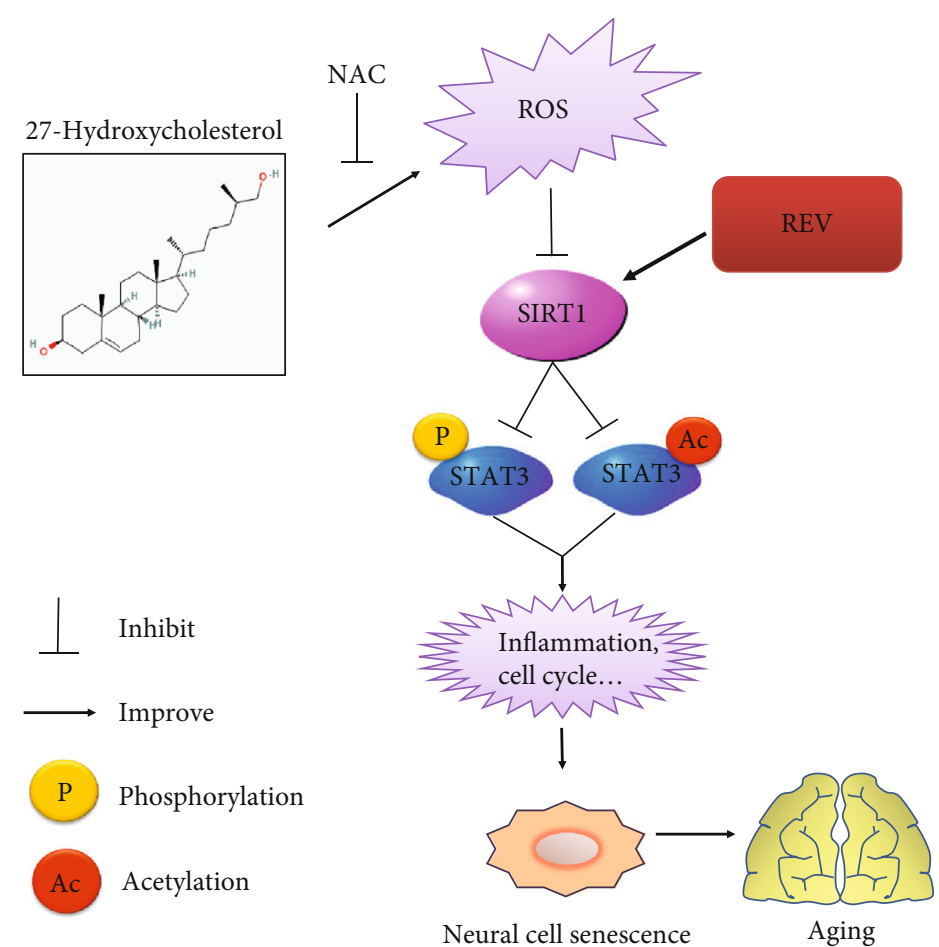

FIGURE 7: Schematic representation of molecular mechanisms involved in 27HC-induced senescence. 27HC induces a high level of ROS generation, which can downregulate the expression of SIRT1 and subsequently activate the phosphorylation and acetylation of STAT3, which is regulated by SIRT1. Activation of STAT3 triggers aging-related changes in inflammation and the cell cycle leading to neural cell senescence and organism aging.

Current studies showed that both STAT3 phosphorylation and STAT3 acetylation play important functions in transcriptional regulation. The role of STAT3 phosphorylation has been well studied, but the research of STAT3 acetylation is relatively new. There are literatures on the regulation between STAT3 phosphorylation and STAT3 acetylation, but it is not clear which is more important. Many studies have suggested that SIRT1 inhibited STAT3 phosphorylation by deacetylating STAT3 $[13,44]$. In the present study, we found knockdown SIRT1, the best characterized member of class III HDACs, which increased the expression of the STAT3 Lys685 site, and further upregulated STAT3 phosphorylation in Tyr705 (Figure 2). Furthermore, we found that REV, a naturally occurring phytoalexin that inhibits STAT3 acetylation [43], weakened the suppressive function of 27HC on SIRT1 and then inhibited the expression of Ac-STAT3 $3^{\text {Lys685 }}$ and pSTAT3 ${ }^{\text {Tyr705 }}$ promoted by $27 \mathrm{HC}$ (Figure 4). Suggesting that STAT3 phosphorylation may regulated by STAT3 acetylation in the cellular senescence induced by $27 \mathrm{HC}$. The mode of regulation between STAT3 phosphorylation and STAT3 acetylation needs to be further clarified.

Here, zebrafish was used as an in vivo model; the results suggested that $27 \mathrm{HC}$ promoted the accumulation of ROS and aging in zebrafish larvae, which differed from the protective effects of $\mathrm{E}_{2}$. Meanwhile, we found that the ROS scavenger NAC and REV markedly blocked the aging induced by $27 \mathrm{HC}$. Furthermore, our results showed that $70 \%$ of the zebrafish larvae in the 27HC-exposed group no longer turned routinely along the inner wall of the 96-well plate compared to the control group, which suggested that $27 \mathrm{HC}$ affects spontaneous locomotor behavior. And REV alleviates the effect of $27 \mathrm{HC}$ via regulating the level of SIRT1 and IL-6.

\section{Conclusions}

In summary, our findings demonstrated that $27 \mathrm{HC}$ induced neural cell senescence and aging in the neural spinal cord of the zebrafish model. The 27HC induced the acetylation of STAT3 via SIRT1, and the 27HC-induced decrease in SIRT1 was associated with ROS-mediated methylation. Moreover, REV attenuated 27HC-induced senescence by inhibiting STAT3 signaling via SIRT1 in neural cells and zebrafish (Figure 7). In conclusion, REV alleviated 27HC-induced senescence in neural cells and affected zebrafish locomotor behavior by activating SIRT1-mediated STAT3 signaling.

\section{Abbreviations}

27HC: $\quad$ 27-Hydroxycholesterol

AZA: Azacitidine

E2: $\quad 17 \beta$-Estradiol

NAC: N-Acetylcysteine

REV: Resveratrol

ROS: $\quad$ Reactive oxygen species

SA- $\beta$-Gal: Senescence-associated $\beta$-galactosidase

SAM: $\quad$ S-Adenosyl methionine

SIRT1: Sirtuin-1

STAT3: $\quad$ Signal transducer and activator of transcription 3. 


\section{Data Availability}

The data used to support the findings of the present study are available from the corresponding authors upon request.

\section{Conflicts of Interest}

The authors declare that they have no conflicts of interest.

\section{Authors' Contributions}

Zhong Li and Ming Zhou participated in the experimental design. Jiao Liu and Kailin Jiao performed the experiments. Qian Zhou, Jun Yang, and Keke Yang contributed to statistical analyses. Jiao Liu, Kailin Jiao, and Chunyan $\mathrm{Hu}$ were responsible for writing the manuscript. Jiao Liu, Kailin Jiao, Qian Zhou, and Jun Yang contributed equally to this work.

\section{Acknowledgments}

This work was supported by the National Natural Science Foundation of China (81673205) and the Center for Global Health, School of Public Health, Nanjing Medical University. This project was also funded by the Priority Academic Program Development of Jiangsu Higher Education Institutions (PAPD).

\section{References}

[1] A. Asghari and M. Umetani, "Obesity and cancer: 27-hydroxycholesterol, the missing link," International Journal of Molecular Sciences, vol. 21, no. 14, p. 4822, 2020.

[2] C. Parrado-Fernandez, V. Leoni, A. Saeed et al., "Sex difference in flux of 27-hydroxycholesterol into the brain," British Journal of Pharmacology, 2021.

[3] C. D. DuSell, M. Umetani, P. W. Shaul, D. J. Mangelsdorf, and D. P. McDonnell, "27-Hydroxycholesterol is an endogenous selective estrogen receptor modulator," Molecular Endocrinology, vol. 22, no. 1, pp. 65-77, 2008.

[4] S. W. Brooks, A. C. Dykes, and B. G. Schreurs, "A highcholesterol diet increases 27-hydroxycholesterol and modifies estrogen receptor expression and neurodegeneration in rabbit hippocampus," Journal of Alzheimer's Disease, vol. 56, no. 1, pp. 185-196, 2016.

[5] J. Liu, Y. Liu, J. Chen et al., "The ROS-mediated activation of IL-6/STAT3 signaling pathway is involved in the 27hydroxycholesterol-induced cellular senescence in nerve cells," Toxicology In Vitro, vol. 45, Part 1, pp. 10-18, 2017.

[6] Z. Shen, D. Zhu, J. Liu et al., "27-Hydroxycholesterol induces invasion and migration of breast cancer cells by increasing MMP9 and generating EMT through activation of STAT-3," Environmental Toxicology and Pharmacology, vol. 51, pp. 18, 2017.

[7] D. Zhu, Z. Shen, J. Liu et al., "The ROS-mediated activation of STAT-3/VEGF signaling is involved in the 27hydroxycholesterol-induced angiogenesis in human breast cancer cells," Toxicology Letters, vol. 264, pp. 79-86, 2016.

[8] D. E. Johnson, R. A. O'Keefe, and J. R. Grandis, "Targeting the IL-6/JAK/STAT3 signalling axis in cancer," Nature Reviews. Clinical Oncology, vol. 15, no. 4, pp. 234-248, 2018.
[9] A. C. Guanizo, C. D. Fernando, D. J. Garama, and D. J. Gough, "STAT3: a multifaceted oncoprotein," Growth Factors, vol. 36, no. 1-2, pp. 1-14, 2018.

[10] H. Lee, A. J. Jeong, and S. K. Ye, "Highlighted STAT3 as a potential drug target for cancer therapy," BMB Reports, vol. 52, no. 7, pp. 415-423, 2019.

[11] N. Fathi, G. Rashidi, A. Khodadadi, S. Shahi, and S. Sharifi, "STAT3 and apoptosis challenges in cancer," International Journal of Biological Macromolecules, vol. 117, pp. 993-1001, 2018.

[12] S. Michels, M. Trautmann, E. Sievers et al., "SRC signaling is crucial in the growth of synovial sarcoma cells," Cancer Research, vol. 73, no. 8, pp. 2518-2528, 2013.

[13] Z. L. Yuan, Y. J. Guan, D. Chatterjee, and Y. E. Chin, "Stat 3 dimerization regulated by reversible acetylation of a single lysine residue," Science, vol. 307, no. 5707, pp. 269-273, 2005.

[14] J. Ni, Y. Shen, Z. Wang et al., "P300-dependent STAT3 acetylation is necessary for angiotensin II-induced pro-fibrotic responses in renal tubular epithelial cells," Acta Pharmacologica Sinica, vol. 35, no. 9, pp. 1157-1166, 2014.

[15] T. Finkel, C. X. Deng, and R. Mostoslavsky, "Recent progress in the biology and physiology of sirtuins," Nature, vol. 460, no. 7255, pp. 587-591, 2009.

[16] J. Lu, L. Zhang, X. Chen et al., "SIRT1 counteracted the activation of STAT3 and NF- $\kappa$ B to repress the gastric cancer growth," International Journal of Clinical and Experimental Medicine, vol. 7, no. 12, pp. 5050-5058, 2014.

[17] S. Liu, Z. Zheng, S. Ji et al., "Resveratrol reduces senescenceassociated secretory phenotype by SIRT1/NF- $\kappa$ B pathway in gut of the annual fish _Nothobranchius guentheri_," Fish \& Shellfish Immunology, vol. 80, pp. 473-479, 2018.

[18] V. Foti Cuzzola, R. Ciurleo, S. Giacoppo, S. Marino, and P. Bramanti, "Role of resveratrol and its analogues in the treatment of neurodegenerative diseases: focus on recent discoveries," CNS \& Neurological Disorders Drug Targets, vol. 10, no. 7, pp. 849-862, 2011.

[19] A. Henn, S. Lund, M. Hedtjärn, A. Schrattenholz, P. Pörzgen, and M. Leist, "The suitability of BV2 cells as alternative model system for primary microglia cultures or for animal experiments examining brain inflammation," ALTEX, vol. 26, no. 2, pp. 83-94, 2009.

[20] K. Mussina, D. Toktarkhanova, and O. Filchakova, "Nicotinic acetylcholine receptors of PC12 cells," Cellular and Molecular Neurobiology, vol. 41, no. 1, pp. 17-29, 2021.

[21] J. Zhang, S. An, W. Hu et al., "The neuroprotective properties of Hericium erinaceus in glutamate-damaged differentiated PC12 cells and an Alzheimer's disease mouse model," International Journal of Molecular Sciences, vol. 17, no. 11, 2016.

[22] A. Saera-Vila, P. E. Kish, and A. Kahana, "Automated scalable heat shock modification for standard aquatic housing systems," Zebrafish, vol. 12, no. 4, pp. 312-314, 2015.

[23] G. Kari, U. Rodeck, and A. P. Dicker, "Zebrafish: an emerging model system for human disease and drug discovery," Clinical Pharmacology and Therapeutics, vol. 82, no. 1, pp. 70-80, 2007.

[24] E. J. Kyzar, C. Collins, S. Gaikwad et al., "Effects of hallucinogenic agents mescaline and phencyclidine on zebrafish behavior and physiology," Progress in Neuro-Psychopharmacology \& Biological Psychiatry, vol. 37, no. 1, pp. 194-202, 2012. 
[25] L. Grossman, E. Utterback, A. Stewart et al., "Characterization of behavioral and endocrine effects of LSD on zebrafish," Behavioural Brain Research, vol. 214, no. 2, pp. 277-284, 2010.

[26] G. P. Dimri, X. Lee, G. Basile et al., "A biomarker that identifies senescent human cells in culture and in aging skin in vivo," Proceedings of the National Academy of Sciences of the United States of America, vol. 92, no. 20, pp. 9363-9367, 1995.

[27] X. Wang, F. Jiang, J. Mu et al., “Arsenic trioxide attenuates the invasion potential of human liver cancer cells through the demethylation-activated microRNA-491," Toxicology Letters, vol. 227, no. 2, pp. 75-83, 2014.

[28] H. M. O'Hagan, W. Wang, S. Sen et al., "Oxidative damage targets complexes containing DNA methyltransferases, SIRT1, and polycomb members to promoter CpG Islands," Cancer Cell, vol. 20, no. 5, pp. 606-619, 2011.

[29] J. Van Houcke, L. De Groef, E. Dekeyster, and L. Moons, “The zebrafish as a gerontology model in nervous system aging, disease, and repair," Ageing Research Reviews, vol. 24, Part B, 2015.

[30] D. D. Zhang, H. L. Yu, W. W. Ma et al., "27-Hydroxycholesterol contributes to disruptive effects on learning and memory by modulating cholesterol metabolism in the rat brain," Neuroscience, vol. 300, pp. 163-173, 2015.

[31] G. Marwarha and O. Ghribi, "Does the oxysterol 27hydroxycholesterol underlie Alzheimer's disease- Parkinson's disease overlap?," Experimental Gerontology, vol. 68, pp. 13$18,2015$.

[32] L. Bonfili, V. Cecarini, M. Cuccioloni et al., "SLAB51 probiotic formulation activates SIRT1 pathway promoting antioxidant and neuroprotective effects in an AD mouse model," Molecular Neurobiology, vol. 55, no. 10, pp. 7987-8000, 2018.

[33] C. Moussa, M. Hebron, X. Huang et al., "Resveratrol regulates neuro-inflammation and induces adaptive immunity in Alzheimer's disease," Journal of Neuroinflammation, vol. 14, no. 1, p. 1, 2017.

[34] P. Ding, D. Ren, S. He et al., "Sirt 1 mediates improvement in cognitive defects induced by focal cerebral ischemia following hyperbaric oxygen preconditioning in rats," Physiological Research, vol. 66, no. 6, pp. 1029-1039, 2017.

[35] R. H. Houtkooper, E. Pirinen, and J. Auwerx, "Sirtuins as regulators of metabolism and healthspan," Nature Reviews. Molecular Cell Biology, vol. 13, no. 4, pp. 225-238, 2012.

[36] Z. Zou, B. Liu, L. Zeng et al., " $\mathrm{Cx} 43$ inhibition attenuates sepsis-induced intestinal injury via downregulating ROS transfer and the activation of the JNK1/Sirt 1/fox O3a signaling pathway," Mediators of Inflammation, vol. 2019, Article ID 7854389, 13 pages, 2019.

[37] M. Yamakuchi, M. Ferlito, and C. J. Lowenstein, "miR-34a repression of SIRT1 regulates apoptosis," Proceedings of the National Academy of Sciences of the United States of America, vol. 105, no. 36, pp. 13421-13426, 2008.

[38] Z. Shen, K. Jiao, M. Teng, and Z. Li, “Activation of STAT-3 signalling by RECK downregulationviaROS is involved in the 27hydroxycholesterol-induced invasion in breast cancer cells," Free Radical Research, vol. 54, no. 2-3, pp. 126-136, 2020.

[39] B. A. Gomes, J. P. Silva, C. F. Romeiro et al., "Neuroprotective Mechanisms of Resveratrol in Alzheimer's Disease: Role of SIRT1," vol. 2018, Article ID 8152373, Oxidative medicine and cellular longevity, 2018.
[40] X. Hou, D. Rooklin, H. Fang, and Y. Zhang, "Resveratrol serves as a protein-substrate interaction stabilizer in human SIRT1 activation," Scientific Reports, vol. 6, 2016.

[41] L. X. Wang, A. Heredia, H. Song et al., "Resveratrol glucuronides as the metabolites of resveratrol in humans: characterization, synthesis, and anti-HIV activity," Journal of Pharmaceutical Sciences, vol. 93, no. 10, pp. 2448-2457, 2004.

[42] M. Eufemi, R. Cocchiola, D. Romaniello et al., "Acetylation and phosphorylation of STAT3 are involved in the responsiveness of microglia to beta amyloid," Neurochemistry International, vol. 81, pp. 48-56, 2015.

[43] S. Li, W. Zhang, Y. Yang et al., "Discovery of oral-available resveratrol-caffeic acid based hybrids inhibiting acetylated and phosphorylated STAT3 protein," European Journal of Medicinal Chemistry, vol. 124, pp. 1006-1018, 2016.

[44] R. Sestito, S. Madonna, C. Scarponi et al., "STAT3-dependent effects of IL-22 in human keratinocytes are counterregulated by sirtuin 1 through a direct inhibition of STAT3 acetylation," The FASEB Journal, vol. 25, no. 3, pp. 916-927, 2011. 